\title{
Principal Symmetric Meshes
}

\author{
DAVIDE PELLIS ${ }^{*}$, TU Wien \\ HUI WANG ${ }^{*}$, DUT \\ MARTIN KILIAN, TU Wien \\ FLORIAN RIST, KAUST, TU Wien \\ HELMUT POTTMANN, KAUST, TU Wien \\ CHRISTIAN MÜLLER, TU Wien
}
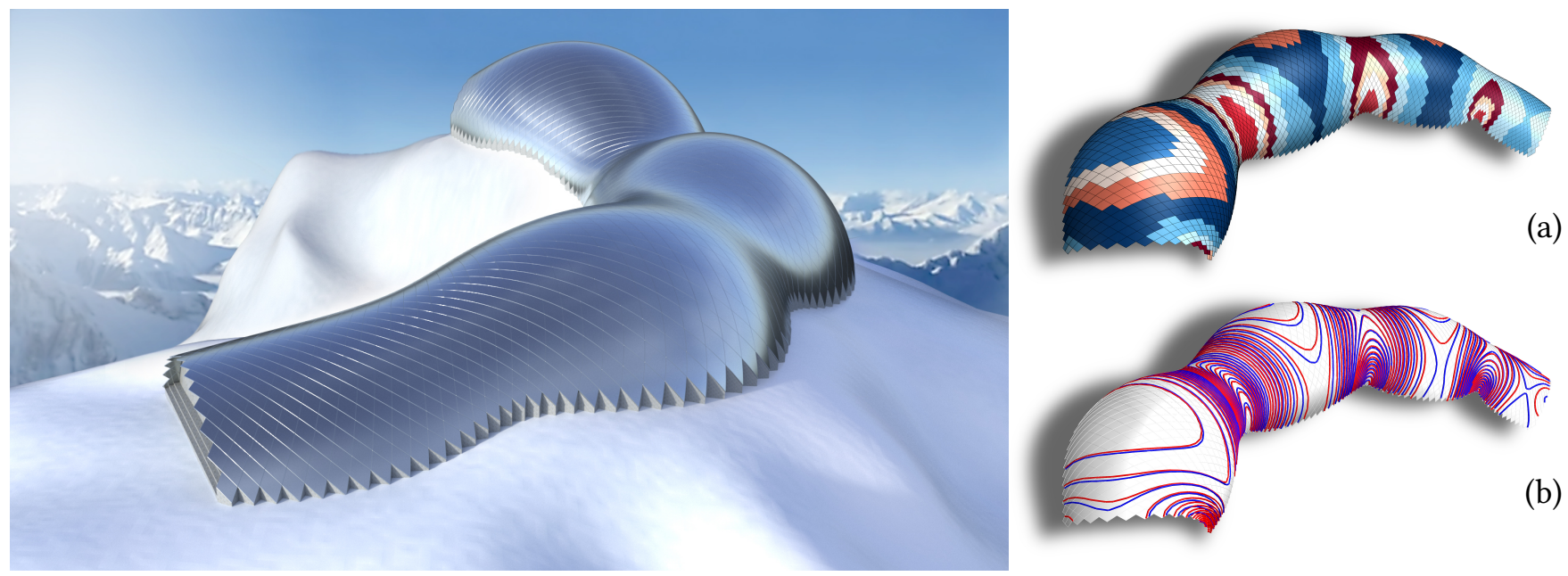

Fig. 1. Architectural design of a modern mountain leisure and tourism center. The geometric shape of the hull is a discrete principal symmetric parametrization (an S-net) of a Weingarten surface of type $a \kappa_{1}+b \kappa_{2}=c$ (see Sec. 3.5). The big advantage lies in the fabrication of its doubly curved panels by a substantial reduction of necessary molds, as the same mold can be used along curves of constant curvature illustrated by isolines (b). Panels with identical molds are clustered together and highlighted by the same color (a). The network of this S-net assumes constant intersection angles which contributes to its aesthetic.

The isolines of principal symmetric surface parametrizations run symmetrically to the principal directions. We describe two discrete versions of these special nets/quad meshes which are dual to each other and show their usefulness for various applications in the context of fabrication and architectural design. Our discretization of a principal symmetric mesh comes naturally with a family of spheres, the so-called Meusnier and Mannheim spheres. In our representation of principal symmetric meshes, we have direct control over the radii of theses spheres and the intersection angles of the parameter lines. This facilitates tasks such as generating Weingarten surfaces including constant mean curvature surfaces and minimal surfaces. We illustrate the potential of Weingarten surfaces for paneling doubly curved freeform

*Joint first authors

Authors' addresses: Davide Pellis, TU Wien; Hui Wang, DUT; Martin Kilian, TU Wien; Florian Rist, KAUST, TU Wien; Helmut Pottmann, KAUST, TU Wien; Christian Müller, TU Wien.

Permission to make digital or hard copies of all or part of this work for personal or classroom use is granted without fee provided that copies are not made or distributed for profit or commercial advantage and that copies bear this notice and the full citation on the first page. Copyrights for components of this work owned by others than ACM must be honored. Abstracting with credit is permitted. To copy otherwise, or republish, to post on servers or to redistribute to lists, requires prior specific permission and/or a fee. Request permissions from permissions@acm.org.

(c) 2020 Association for Computing Machinery.

0730-0301/2020/7-ART127 \$15.00

https://doi.org/10.1145/3386569.3392446 facades by significantly reducing the number of necessary molds. Moreover, we have direct access to curvature adaptive tool paths for cylindrical CNC milling with circular edges as well as flank milling with rotational cones. Furthermore, the construction of curved support structures from congruent circular strips is easily managed by constant sphere radii. The underlying families of spheres are in a natural way discrete curvature spheres in analogy to smooth Möbius and Laguerre geometry which further leads to a novel discrete curvature theory for principal symmetric meshes.

CCS Concepts: • Computing methodologies $\rightarrow$ Shape modeling; Optimization algorithms.

Additional Key Words and Phrases: discrete differential geometry, architectural geometry, computational fabrication, paneling, normal curvature, sphere geometries, curvature adaptive milling

\section{ACM Reference Format:}

Davide Pellis, Hui Wang, Martin Kilian, Florian Rist, Helmut Pottmann, and Christian Müller. 2020. Principal Symmetric Meshes. ACM Trans. Graph. 39, 4, Article 127 (July 2020), 17 pages. https://doi.org/10.1145/3386569. 3392446

\section{INTRODUCTION}

Meshes are omnipresent in geometric computing: as a geometry representation, as a basis for simulations, and for shape optimization. 
In those cases, meshes are not visible in the final product and typical quality measures include approximation accuracy and the size and shape of faces. However, in certain applications, such as architecture, meshes constitute an important part of the design and are closely tied to the fabrication process. This leads to constraints related to aesthetics, planarity of faces, the supporting structure along the edges, statics and others.

The present paper contributes to the latter type of meshes. We present a so far largely neglected class of quad meshes which not only represent the shape of the surface, but are motivated by aspects of aesthetics and fabrication. These meshes have the capability to make simple shapes more interesting. The Swiss Re Tower (Fig. 2, left) provides a good example. The shape of the tower is a simple rotational surface which would look rather boring if it had been paneled along floor slabs (parallel circles) and vertical profile curves, i.e., along principal curvature lines of the rotational surface. However, the architects chose a more interesting solution: It follows directions which are symmetric with respect to the principal directions, but they are not following principal directions themselves. In other words, these meshes discretize a network of curves on a surface whose bisecting directions in each point are the principal curvature directions. We call them principal symmetric (ps) meshes.

Since the asymptotic directions on negatively curved surfaces (directions of vanishing normal curvature) are symmetric with respect to principal directions, the network of asymptotic curves on a surfaces is a smooth ps net on a surface. The most widely used discretization is that of A-nets, which are quad meshes with planar vertex stars: the four edges emanating from a vertex lie in a plane [Bobenko and Suris 2008].

Already Finsterwalder [1899] pointed to a simple way of building a surface model based on asymptotic curves: Finsterwalder builds the model from straight and originally flat strips of material which is easily bent but not stretched (paper, cardboard, sheet metal). Such a strip can be attached orthogonally to a reference surface $S$ if and only if it follows an asymptotic curve of $S$. This allows one to build curved structures from straight strips, as beautifully demonstrated in the asymptotic gridshells of E. Schling [2018] (Fig. 2).

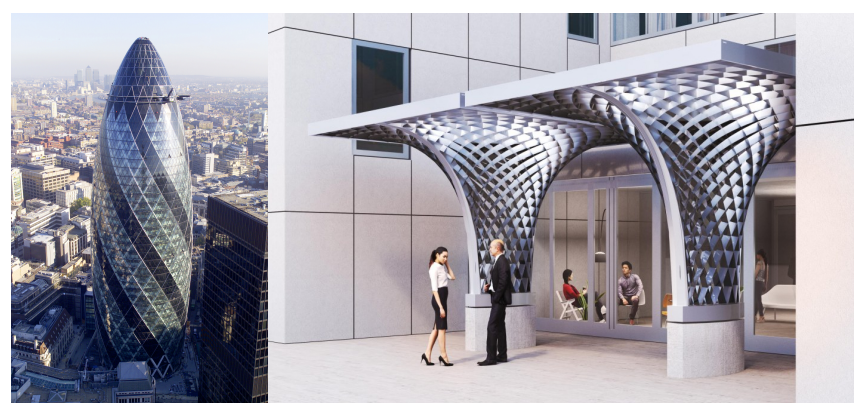

Fig. 2. Principal symmetric meshes in architecture. Left: Swiss Re Tower, London, by N. Foster and K. Shuttleworth, Right: Canopy for a hotel in Ingolstadt, Germany, by E. Schling and J. Schikore.

Asymptotic gridshells can be generalized to surface models which are fabricated from planar circular strips of constant radius [Schling et al. 2018]. In these structures the curved strips are arranged along curves of constant normal curvature on a reference surface. Directions with the same normal curvature are symmetric with respect to the principal directions and therefore one again arrives at ps meshes. In [Schling et al. 2018], the ps property is expressed via S-nets. These are quad meshes with spherical vertex stars, meaning that a vertex and its four connected neighboring vertices lie on a sphere. However, the paper does not go much beyond the definition of S-nets and thus just scratches the surface of an interesting topic, which we will now elaborate in much more depth and illustrate with several quite different applications.

\subsection{Overview and contributions}

The main goal of the present paper is a careful study of the geometry of principal symmetric (ps) meshes and their applications in computational design and fabrication:

- It turns out that ps curve networks on a surface are objects of sphere geometries. Hence, we study ps meshes within the two major and practically most relevant sphere geometries, namely Möbius geometry (S-nets mentioned above) and Laguerre geometry (leading to the new $S^{*}$-nets).

- We discuss the design space of modeling S-nets which is quite large: for any freely chosen one-parameter family of curves covering a designed reference surface there exists a unique transversal family which complements it to an $S$-net.

- We develop a novel discrete curvature theory for S-nets based on a discrete Gauss map.

- The wide class of ps meshes contains a number of remarkable special cases. These include various classes of so-called Weingarten surfaces, which are surfaces whose principal curvatures $\kappa_{1}, \kappa_{2}$ satisfy an equation $F\left(\kappa_{1}, \kappa_{2}\right)=0$. Weingarten surfaces possess only a one-parameter family of curvature elements. We show how one can use this for architectural paneling solutions with curved panels. For $N$ panels, one needs only roughly $\sqrt{N}$ molds.

- We show how to compute ps meshes via numerical optimization. In particular we show how to (i) approximate a given surface with a ps mesh and (ii) given the combinatorics of a quad mesh, we can optimize for the ps property, incorporating additional constraints. This allows us to compute interesting geometry (e.g., Weingarten surfaces) from combinatorics.

- We show that S-nets provide all information needed for curvature adaptive $\mathrm{CNC}$ machining and demonstrate the high quality surface finish one can achieve in this way.

- While meshes which follow principal directions are easily optimized for planarity of their faces (for circular and conical meshes, the definition even includes planarity of faces), this is not true for ps meshes. We show how and under which circumstances one can obtain practically useful ps meshes with planar quads.

\subsection{Related Work}

Quad mesh design and in particular quad-remeshing is a highly popular topic in geometry processing. It is far beyond the scope of our paper to provide a brief overview. Hence, we refer to the survey [Bommes et al. 2013] and point to a few recent contributions [Diamanti et al. 2015; Jakob et al. 2015; Sageman-Furnas et al. 
2019; Vaxman et al. 2017] that present important concepts and algorithms for the alignment of a quad mesh along prescribed directions. Remeshing is not a main target of our work and largely left for future research, but ideas in those papers could be important to provide efficient tools for the design of ps meshes on a given surface.

In discrete differential geometry (DDG), most research on quad mesh based surfaces has focused on those with planar faces (conjugate parameterizations) or planar vertex stars (asymptotic parameterizations) [Bobenko and Suris 2008]. An exception is the work on edge-constraint nets [Hoffmann et al. 2017]. We will further contribute to this class of discrete surfaces and show that S-nets with vertex spheres of constant radius are edge-constraint nets.

While a general discussion of ps meshes is new to the present paper, there are special cases in which they appear in the literature. To the best of our knowledge the earliest occurrence is in [Wunderlich 1951], where special S-nets arise as offsets of discrete surfaces of constant negative Gaussian curvature. The sphere condition for S-nets and its dual counterpart, implied on diagonal nets, has been used to characterize discrete isothermic surfaces and their Laguerre geometric counterparts [Bobenko and Suris 2006]. Certain ps triangle meshes recently appeared in a study of polyhedral surfaces with high visual smoothness [Pellis et al. 2019].

The two types of ps meshes which we are studying are distinguished by the sphere geometry to which they belong. Recall that the two major classes of meshes which follow principal directions are also classified in terms of sphere geometries: circular meshes (Möbius) and conical meshes (Laguerre) [Bobenko and Suris 2008]. There have also been contributions to geometry processing which operate within sphere geometries, e.g. [Bobenko and Schröder 2005; Vaxman et al. 2015, 2018], or concern meshes of spheres [Thiery et al. 2013, 2016; Tkach et al. 2016].

A main aspect in our work is its relation between discrete surface models and specific applications in architecture and fabrication. A substantial early contribution to this area has been made by S. Finsterwalder [1899], who presented numerous ideas for the fabrication of surfaces from strips, on their deformation and on shapes in force equilibrium. Much later, with the advent of freeform architecture, the construction of surfaces from simple elements became again interesting and in fact a big challenge. This led to research on architectural geometry, which we do not aim to review here, but just refer to the survey [Pottmann et al. 2015]. The beautiful interplay between DDG and fabrication is seen in a number of recent contributions. This includes research on Chebyshev nets and wire meshes [Garg et al. 2014; Sageman-Furnas et al. 2019], on discrete orthogonal geodesics and developable surfaces [Rabinovich et al. 2018a,b; Wang et al. 2019], or on conformal maps and certain types of auxetic materials [Konaković et al. 2016, 2018].

\section{S-NETS AND S*-NETS}

Our goal is to discretize smooth $S$-nets, i.e., surface parametrizations where both parameter lines (isolines) are symmetric with respect to the principal directions (cf. Def. 2.1). However, before we enter the road of discretization let us briefly recall the related notions from smooth differential geometry. We will compare the classical notions of Meusnier spheres and Euler's formula with the lesser known
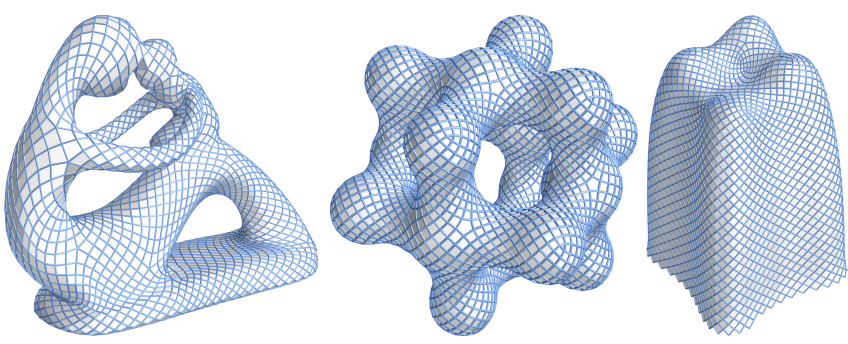

Fig. 3. We obtain discrete S-nets by applying our algorithm to an initial mesh with edge polylines following a cross field whose axes are symmetric to the principal directions of an underlying reference surface. Even though remeshing typical computer graphics models into ps nets is not our primary goal these examples illustrate the generality and flexibility of our method. In this way, the Lilium tower (right), as originally planned with a mesh aligned with the story levels, assumes another interesting appearance.

Mannheim sphere and Blaschke's formula, which are dual to each other. Both settings deal with the notion of the normal curvature of surfaces and lead to novel discretizations of S-nets.

\subsection{Smooth setting}

In our paper, the notion of the normal curvature is key. We therefore first recall the definition and properties of the classical normal curvature before we then look at its dual counterpart. Afterwards we will have a brief look at smooth S-nets and discuss their d.o.f.

2.1.1 Normal curvature. Let us consider a smooth surface $S$ with a tangent vector $t$ attached to it at a surface point $p \in S$. The plane $\tau$ spanned by the tangent vector $t$ and the surface normal $n$ at $p$ intersects the surface $S$ (in general at least locally) along a curve $c$ (see Figure 4 left). Now, the normal curvature $\kappa_{n}$ of $S$ in direction $t$ is defined as the curvature of the intersection curve $c$ at $p$ (see, e.g., [do Carmo 1976, p. 142]). On the other hand, the orthogonal projection onto plane $\tau$ of any other arbitrary curve $\tilde{c} \subset S$ passing through $p$ and having the same tangent $t$ yields a projected curve with the same curvature (theorem of Meusnier, cf. e.g., [do Carmo 1976, p. 142]). In that sense we can measure the normal curvature of any curve $\tilde{c}$ since it only depends on the tangent.

As we rotate the tangent $t$ in the tangent plane about $p$ we obtain different intersection curves and therefore different normal curvatures $\kappa_{n}$. The maximal normal curvature $\kappa_{1}$ and the minimal normal curvature $\kappa_{2}$ are the principal curvatures at $p$ [do Carmo 1976, p. 144]. The two directions $e_{1}, e_{2}$ corresponding to the two principal curvatures are orthogonal directions, the so called principal directions [do Carmo 1976, p. 144].

2.1.2 Parametrizations and smooth nets. Let $S$ be a surface with parametrization $f: U \subseteq \mathbb{R}^{2} \rightarrow \mathbb{R}^{3}$, i.e., $S=f(U)$, and with unit normal vector field or Gauss map $n$.

Definition 2.1. We call a smooth parametrization a smooth S-net if the two tangents to the parameter lines at each point are symmetric with respect to the principal directions.

Let us denote the coordinate functions of the first fundamental form by $E=\left\langle f_{u}, f_{u}\right\rangle, F=\left\langle f_{u}, f_{v}\right\rangle, G=\left\langle f_{v}, f_{v}\right\rangle$, and of the second fundamental form by $e=\left\langle f_{u u}, n\right\rangle, f=\left\langle f_{u v}, n\right\rangle, g=\left\langle f_{v v}, n\right\rangle$. Then 

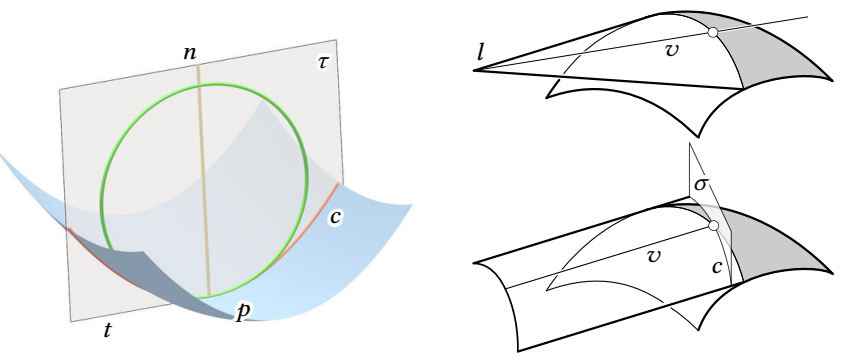

Fig. 4. Left: Normal curvature. The plane spanned by a tangent $t$ and the surface normal $n$ at a surface point $p$ intersects the surface along a curve $c$. The curvature $\kappa_{n}$ of that curve at $p$ is the normal curvature. Top-Right: A tangential cone. A flashlight $l$ positioned at a surface tangent $v$ generates a cone of light rays which touch the surface along the shadow contour. This cone (interpreted as the envelope of planes through point $l$ ) is the dual analogue to an intersection curve (left) with a plane (interpreted as the points on a plane). Bottom-Right: A tangential cylinder. The flashlight positioned at infinity generates a cylinder of light rays which touch the surface along the shadow contour. This cylinder is dual to the intersection curve with the normal plane $\tau$ (left). By the name dual curvature radius $\rho_{n}^{*}$ we call the curvature radius of the intersection curve $c$ of the plane $\sigma$ which is orthogonal to the rulings of this cylinder. Note that the tangent of $c$ is (in general) different from the tangent of the shadow contour.

the normal curvature in direction $t=a f_{u}+b f_{v}$ can be expressed in terms of the coordinate functions of the fundamental forms by (see, e.g.,[do Carmo 1976, p. 142])

$$
\kappa_{n}=\frac{a^{2} e+2 a b f+b^{2} g}{a^{2} E+2 a b F+b^{2} G} .
$$

Consequently, the normal curvature in the direction of $f_{u}$ (i.e., $a=1, b=0)$ is $e / E$ and analogously in the direction of $f_{v}$ is $g / G$. A characterization of $f$ being a smooth S-net is therefore $e G=E g$. The proofs of the following lemma can be found in Appendix A.

LEMMA 2.2. Apart from umbilical points an S-net is the same as a net with equal normal curvature in both directions.

Lemma 2.3. Smooth S-nets are Möbius invariant, which means that any Möbius transformations maps an S-net to an S-net.

Proof. Principal parametrizations, i.e., parameter lines following the principal directions, are Möbius invariant (see, e.g., [Blaschke 1929]). And since Möbius transformations are angle preserving, any curve network that is symmetric to the principal directions stays that way under Möbius transformations.

2.1.3 Gauss map of an $S$-net. In the basis of the principal directions $e_{1}, e_{2}$ the two symmetric tangent vectors of an S-net are of the form $f_{u} /\left\|f_{u}\right\|=a e_{1}+b e_{2}$ and $f_{v} /\left\|f_{v}\right\|=a e_{1}-b e_{2}$. The partial derivatives on the Gauss map $n=\frac{f_{u} \times f_{v}}{\left\|f_{u} \times f_{v}\right\|}$ are also symmetric with respect to $e_{1}$ because $n_{u}$ is parallel to $-\kappa_{1} a e_{1}-\kappa_{2} b e_{2}$ and $n_{v}$ is parallel to $-\kappa_{1} a e_{1}+\kappa_{2} b e_{2}$.

A consequence is the following offsetting property: all parallel surfaces $f^{d}=f+d n$ of a smooth S-net $f$ are smooth S-nets since the Gauss map of each offset surface $f^{d}$ is the same $n$ and the principal directions do not change either.

Classical and simple examples of S-nets include the following:
- Take an arbitrary space curve $c$ and rotate it around an axis. The complementary family is obtained by reflecting $c$ in a meridian plane (plane through the axis) and rotate this curve as well.

- Diagonal nets of isothermic parametrizations (i.e., conformal curvature line parametrizations).

- Asymptotic nets.

- Möbius transformations applied to the above mentioned nets.

2.1.4 Dual viewpoint. The notion of duality in what follows is not the duality of projective geometry in a strict sense, but the concepts are still very closely related. The basic idea of duality in 3D geometry is to formulate equivalent statements by exchanging the notions "point" and "plane". So instead of considering a curve on a surface as "collection of points", in our dual viewpoint we consider a curve to be the curve of tangency of a "collection of tangent planes" along that curve. Thus the intersection curve of a surface with a plane through a tangent line is transferred to the tangential cone of a surface with the vertex lying on a tangent line (like the enveloping light rays of a flashlight; see Figure 4 top-right).

The particular case in the dual setting where the vertex of the cone is a point at infinity and therefore degenerating the tangential cone to a tangential cylinder is special and important (Fig. 4 bttm.-right).

Definition 2.4. We call the curvature radius of the intersection curve of that cylinder with a plane orthogonal to the rulings the dual curvature radius $\rho_{n}^{*}$.

2.1.5 Dupin indicatrix. The Dupin indicatrix (see, e.g., [do Carmo 1976, p. 148]) illustrates the curvature behavior in the tangent plane of a surface point (Fig. 5). It is the set of points $\left|\kappa_{n}\right|^{-1 / 2}\left(\cos \theta e_{1}+\right.$ $\left.\sin \theta e_{2}\right)$, where $\theta$ measures the angle between the first principal direction $e_{1}$ and the direction along which $\kappa_{n}$ is the normal curvature. Dupin indicatrices can be ellipses $\left(\kappa_{1} \kappa_{2}>0\right)$, a pair of hyperbolas $\left(\kappa_{1} \kappa_{2}<0\right)$, or a pair of parallel lines $\left(\kappa_{1} \kappa_{2}=0\right)$.

The square root of the dual curvature radius $\rho_{n}^{*}$ appears as distance between the center and the tangent line which itself has the direction of the ruling of the enveloping developable. This property can be verified by combining Equations (2) and (3) after replacing $\theta$ by $\eta$ in Equation (3), where $\tan \theta \tan \eta=\kappa_{1} / \kappa_{2}$ (since the two corresponding directions are conjugate to each other).

Due to the nature of duality many properties, objects and methods in both settings are similar. We therefore arranged the two columns of the following page mirror symmetric by putting side by side analogous contents.

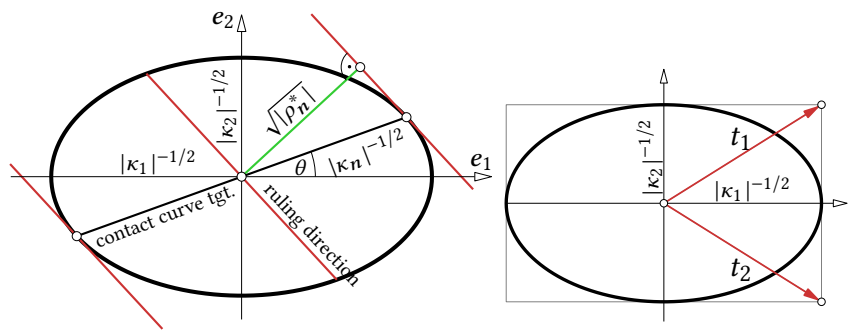

Fig. 5. Dupin indicatrix. Right: The so called characteristic conjugate directions are given by $t_{1}=\left|\kappa_{1}\right|^{-1 / 2} e_{1}+\left|\kappa_{2}\right|^{-1 / 2} e_{2}$ and $t_{2}=\left|\kappa_{1}\right|^{-1 / 2} e_{1}-\left|\kappa_{2}\right|^{-1 / 2} e_{2}$. 
2.1.6 Euler's formula. The formula that relates the normal curvature $\kappa_{n}$ of curves on a surface to the principal curvatures is Euler's formula.

Proposition 2.5 (Euler formula [Do Carmo 1976, P. 145]). Let $t$ be a tangent vector and let $\theta$ denote the angle between $t$ and the first principal direction $e_{1}$. Then the normal curvature $\kappa_{n}$ in the direction $t$ is calculated as

$$
\kappa_{n}=\kappa_{1} \cos ^{2} \theta+\kappa_{2} \sin ^{2} \theta .
$$

The following immediate consequence of Euler's formula is very important for our purposes. It can be easily verified by inserting $\theta$ and $-\theta$ into Euler's formula.

Corollary 2.6. Any two directions $s, t$ in the tangent plane which are symmetric to the principal directions yield the same normal curvatures, i.e., $\kappa_{n}(s)=\kappa_{n}(t)$.

2.1.7 Meusnier sphere. The notions and results of this section can be found, e.g., in [Blaschke and Leichtweiß 1973]. For each direction $t$ at a point $p$ in the tangent plane of a surface with corresponding normal curvature $\kappa_{n}$ the sphere with radius $\frac{1}{\kappa_{n}}$ and center $p+\frac{1}{\kappa_{n}} n$ is called Meusnier sphere. This Meusnier sphere contains the curvature information of any curve that has the same tangent $t$ (see Figure 6 left) as shown by the following proposition. Recall that the osculating plane of a curve $c$ at a point $p$ is the best approximating plane of that curve in $p$. The osculating plane contains the tangent line at $p$ and the osculating circle at $p$.
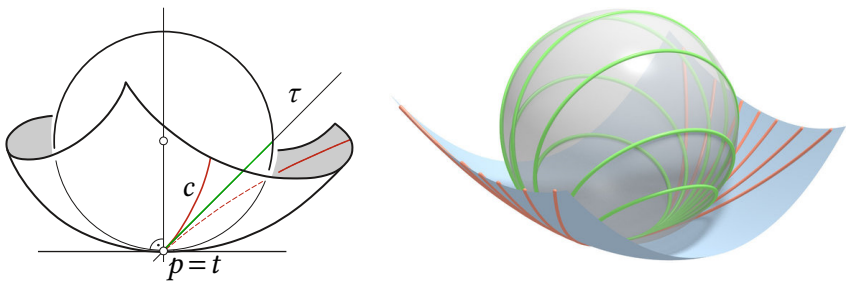

Fig. 6. Meusnier sphere. Left: The red curve $c$ on the surface has a tangent $t$ at $p$ which, in this projection, appears as a point and coincides with $p$. The curve $c$ appears in this projection therefore with a cusp at $p$. Consequently, the osculating plane $\tau$ appears as a line. The Meusnier sphere is the sphere in tangential contact with the surface at $p$ and has radius $1 / \kappa_{n}$, where $\kappa_{n}$ is the normal curvature corresponding to the tangent $t$. The osculating circle of $c$ is the intersection of $\tau$ with the Meusnier sphere. Right: The osculating circles of all curves on a surface that have the same tangent lie on the Meusnier sphere.

Proposition 2.7. Let $S$ be a surface. The osculating circles of all curves $c$ on $S$ which pass through the same point $p \in S$ with the same tangent $t$, arise as intersections of their osculating plane $\tau$ (which passes through $t \subset \tau$ ) with the Meusnier sphere to $(p, t)$ (see also Figure 6).

This characterization of a Meusnier sphere as the union of all curvature circles of curves on a surface with the same tangent (see Figure 6 right) is key in our first discretization (Definition 2.14) of ps nets.
2.1.8 Blaschke formula. The formula that relates the dual curvature radii $\rho_{n}^{*}$ of normal sections of enveloping developables to the principal curvatures is Blaschke's formula.

Proposition 2.8 (BlaschKe Formula [BlaschKe 1916, p. 118]). Let $e$ be a tangent vector and let $\theta$ denote the angle between $e$ and the first principal direction $e_{1}$. Then the dual curvature radius $\rho_{n}^{*}$ corresponding to ruling $e$ is calculated as

$$
\rho_{n}^{*}=\kappa_{2}^{-1} \cos ^{2} \theta+\kappa_{1}^{-1} \sin ^{2} \theta .
$$

The following immediate consequence of Blaschke's formula is very important for our purposes. It can be easily verified by inserting $\theta$ and $-\theta$ into Blaschke's formula.

Corollary 2.9. Any two directions $e, f$ in the tangent plane which are symmetric to the principal directions yield the same dual curvature radii, i.e., $\rho_{n}^{*}(e)=\rho_{n}^{*}(f)$.

2.1.9 Mannheim sphere. The notions and results of this section can be found, e.g., in [Blaschke and Leichtweiß 1973]. For each ruling $e$ at a point $p$ in the tangent plane of a surface with corresponding dual normal curvature radius $\rho_{n}^{*}$ the sphere with radius $\rho_{n}^{*}$ and center $p+$ $\rho_{n}^{*} n$ is called Mannheim sphere. This Mannheim sphere contains the curvature information of any enveloping developable that contains the same ruling $e$ (see Figure 7 left) as shown by the following proposition. Recall that the osculating cone of a developable surface is the best approximating cone with its vertex $v$ on the curve of regression.
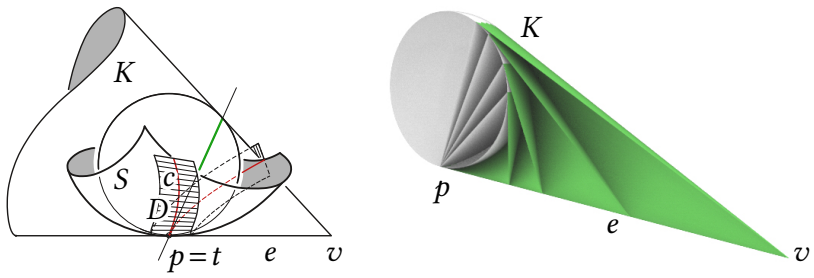

Fig. 7. Mannheim sphere. Left: The red curve $c$ on the surface $S$ has a tangent $t$ at $p$ which, in this projection, appears as a point and coincides with $p$. There is an enveloping developable $D$ in tangential contact with $S$ along $c$ containing the ruling $p v$. The inverse of the curvature of the intersection curve of that enveloping developable $D$ with a plane through $p$ and orthogonal to the ruling $p v$ is the dual curvature radius $\rho_{n}^{*}$. The Mannheim sphere is the sphere in tangential contact with the surface at $p$ and has radius $\rho_{n}^{*}$. The osculating cone $K$ of $D$ is tangentially circumscribed to the Mannheim sphere. Right: The family of osculating cones corresponding to the same tangent envelope the Mannheim sphere.

Proposition 2.10. Let $S$ be a surface. The osculating cones of all enveloping developables along $S$, with the same contact point $p$ and ruling $e$, arise as envelopes of planes which pass through their regression point $v \in e$ and are tangent to the Mannheim sphere to $(p, e)(s e e$ also Figure 7).

This characterization of the Mannheim sphere as envelope of osculating cones of enveloping developables of $S$ with the same contact ruling (see Figure 7 right) is key in our second discretization (Definition 2.17) of ps nets. 
2.1.10 Computing S-net directions. From an algorithmic point of view it is important to compute, for any tangent vector $t$, the tangent vector $u$ that is symmetric to $t$ with respect to the principal directions. The method is provided by the following proposition, whose proof is found in Appendix A.

Proposition 2.11. Let us assume that $f$ is an arbitrary parametrization of a surface and $t=a f_{u}+b f_{v}$ is an arbitrary tangent vector. Then the tangent vector $s=\tilde{a} f_{u}+\tilde{b} f_{v}$ is symmetric to $t$ with respect to the principal directions if and only if

$$
(a, b) M\left(\begin{array}{l}
\tilde{a} \\
\tilde{b}
\end{array}\right):=(a, b)\left(\begin{array}{cc}
2 F e-2 E f & G e-E g \\
G e-E g & 2 G f-2 F g
\end{array}\right)\left(\begin{array}{l}
\tilde{a} \\
\tilde{b}
\end{array}\right)=0,
$$

i.e., if $(a, b)$ is orthogonal to $(\tilde{a}, \tilde{b})$ with respect to the $2 \times 2$ matrix $M$.

2.1.11 Degrees of freedom. So far we did not consider the wealth of possible smooth S-nets on a given surface $f: U \subset \mathbb{R}^{2} \rightarrow \mathbb{R}^{3}$ Suppose we are given a smooth one-parameter family of curves covering the surface. It can be expressed as a family of level set curves $\varphi(u, v)=$ const. covering the parameter domain $U$. Thus the tangent directions to the parameter curves are given by $-\partial_{v} \varphi f_{u}+$ $\partial_{u} \varphi f_{v}$. Consequently, Equation (4) yields a differential equation

$$
\left(-\partial_{v} \varphi, \partial_{u} \varphi\right) M\left(\begin{array}{r}
-\partial_{v} \psi \\
\partial_{u} \psi
\end{array}\right)=0
$$

whose integral curves $\psi(u, v)=$ const. in $U$ trace out a second one-parameter family of curves which together with the family $\varphi(u, v)=$ const. are mapped via $f$ to a smooth S-net (see Figure 8).

Hence, as degrees of freedom for S-net design on a given surface we can prescribe any arbitrary one-parameter family of curves covering that surface and obtain uniquely a second family complementing the given family to a smooth S-net. In the case where a curve touches a principal direction the curve from the second family will also touch the same principal direction generating a local degeneracy where the curves of the two families touch each other. In the same way, as every direction is a principal direction at umbilics, we consider any direction as an S-net tangent at umbilics.

However, in discrete S-nets we do not enforce the sphere condition at irregular vertices but rather enforce fairness around them.
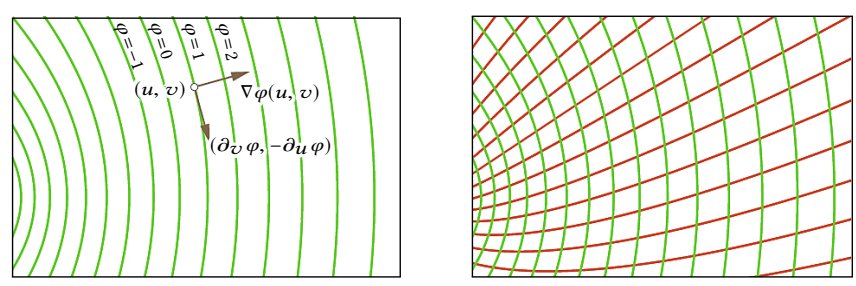

Fig. 8. Left: Isolines of a one-parameter family of curves $\varphi(u, v)=$ const. in the parameter domain $U$. The tangent direction is orthogonal to the gradient hence parallel to $\left(\partial_{v} \varphi,-\partial_{u} \varphi\right)$. Right: The complementary oneparameter family of curves $\psi(u, v)=$ const. (red) which completes the given family (green) to a smooth S-net is governed by the differential equation $\left(-\partial_{v} \varphi, \partial_{u} \varphi\right) M\left(-\partial_{v} \psi, \partial_{u} \psi\right)^{\top}=0$.
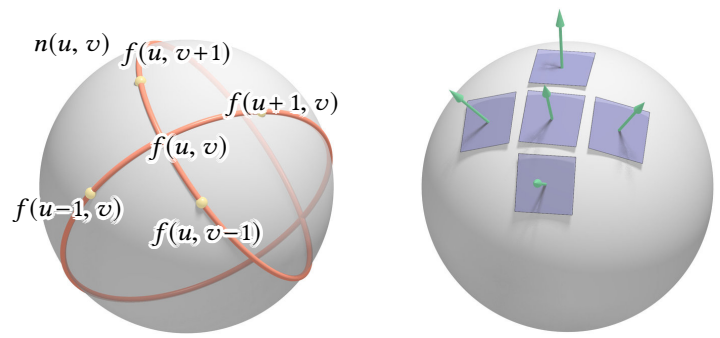

Fig. 9. Left: The two osculating circles determine a discrete normal $n(u, v)$. This normal plus one osculating circle uniquely determines the Meusnier sphere for that direction. In that image both osculating circles lie on the same sphere. Therefore the two corresponding Meusnier spheres coincide and the two normal curvatures are the same which further implies that the two tangent directions are discrete symmetric to the principal directions. Right: Five planes of an $\mathrm{S}^{*}$-net dual to an S-net vertex star (left). The five planes touch a common sphere. Analogously, this common sphere is the Mannheim sphere.

\subsection{Discrete S-nets}

The goal of this section is to give a consistent definition of a discrete S-net that resembles characterizing properties of smooth S-nets.

Let us consider the map $f: \mathbb{Z}^{2} \rightarrow \mathbb{R}^{3}$ representing the vertices of a net. For any parameter line $f(u, \cdot)$ or $f(\cdot, v)$, the circumcircles of three successive vertices $f(u, v-1), f(u, v), f(u, v+1)$ or $f(u-1, v)$, $f(u, v), f(u+1, v)$, determine discrete osculating circles at $f(u, v)$ (see Fig. 9 left).

Tangent vectors to osculating circles. Let us first consider a technical lemma for the description of a tangent vector to a circumcircle whose proof can be found in Appendix A.

Lemma 2.12. Let $A B C$ be a triangle. Then

$$
\frac{B-A}{\|B-A\|^{2}}-\frac{C-A}{\|C-A\|^{2}}
$$

is a tangent vector to the circumcircle of $A B C$ at $A$.

Thus, the tangent of a $u$-parameter line of an S-net is parallel to

$$
\frac{f(u, v)-f(u-1, v)}{\|f(u, v)-f(u-1, v)\|^{2}}+\frac{f(u+1, v)-f(u, v)}{\|f(u+1, v)-f(u, v)\|^{2}} .
$$

There are several possible definitions of normals for discrete nets/meshes, often adapted to the setting one is working in. We choose the following one as it arises natural for S-nets.

Definition 2.13. The discrete normal vector $n(u, v)$ at $f(u, v)$ of an S-net $f$ is given by the unit normal vector that is orthogonal to the tangent vectors of the osculating circles at $f(u, v)$.

Discrete Meusnier sphere. The discrete S-net normal together with one of the two osculating circles determine uniquely a Meusnier sphere and a normal curvature corresponding to that direction. Analogously, the same normal together with the second osculating circle determine uniquely a second Meusnier sphere and a second normal curvature. Now, as our goal is to discretize a curve network symmetric to the principal directions and therefore with the same normal curvature in both directions, we require the two Meusnier spheres to coincide. Or equivalently, the two osculating circles shall lie on 

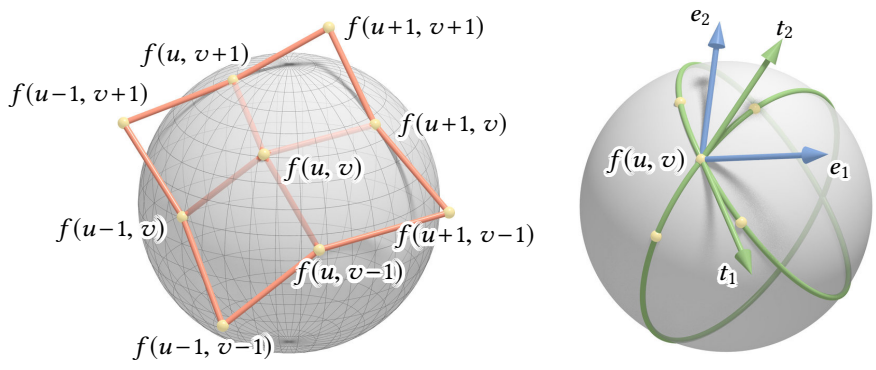

Fig. 10. Left: A vertex star of an S-net. Any central vertex $f(u, v)$ together with its four neighbors $f(u, v+1), f(u, v-1), f(u+1, v), f(u-1, v)$, lie on a common sphere. Right: An S-net vertex star with its two tangent vectors $t_{1}, t_{2}$ which are tangent vectors of the discrete osculating circles. The bisecting vectors $e_{1}, e_{2}$ are the discrete principal directions.

one common sphere (see Figure 9 left). This distinctive property characterizes S-nets.

Definition 2.14. An S-net is a discrete net $f: \mathbb{Z}^{2} \rightarrow \mathbb{R}^{3}$ such that for any $(u, v) \in \mathbb{Z}^{2}$ the five points $f(u, v), f(u, v+1), f(u, v-1)$, $f(u+1, v), f(u-1, v)$, lie on a common sphere (see Figure 10 left).

Since Möbius transformations are sphere preserving, the property of smooth S-nets being Möbius invariant (Lemma 2.3) naturally carries over to the discrete setting as the defining properties only depend on the vertices of each vertex star lying on its own sphere. We record this important fact in the following Lemma.

\section{Lemma 2.15. Discrete S-nets are Möbius invariant.}

Definition 2.16. The equal discrete normal curvatures $\kappa_{n}$ corresponding to both directions of the two tangents of the osculating circles of an S-net are defined to be the inverse radius of their common vertex sphere. The sphere itself is called discrete Meusnier sphere. The discrete principal directions are identified as angle bisectors of the tangent vectors to the osculating circles.

The meshes that we work with have largely $\mathbb{Z}^{2}$ combinatorics, but we allow for irregular vertices and faces at isolated places.

\subsection{Discrete $\mathrm{S}^{*}$-nets}

Discrete $S^{*}$-nets are dual to discrete S-nets in the sense of Section 2.1.4. Instead of a vertex star of five vertices lying on a sphere we are now dealing with five oriented planes in tangential oriented contact with an oriented sphere (i.e., a sphere with oriented normals; outwards or inwards).

Definition 2.17. An $S^{*}$-net is a discrete net $\phi: \mathbb{Z}^{2} \rightarrow$ \{oriented planes in $\left.\mathbb{R}^{3}\right\}$ such that for any $(u, v) \in \mathbb{Z}^{2}$ the five planes $\phi(u, v)$, $\phi(u, v+1), \phi(u, v-1), \phi(u+1, v), \phi(u-1, v)$, are in tangential contact with a common oriented sphere (see Figure 9 right).

Note that the planes of an $S^{*}$-net do not form a planar quadrilateral net since four adjacent planes $f(u, v), f(u+1, v), f(u+1, v+1), f(u, v+1)$ do not meet in one point. The image aside illustrates the planes together with the contact points of neighboring spheres as each plane touches five spheres.
Laguerre geometry is the geometry of oriented spheres and oriented planes. Laguerre transformations operate on those elements in such a way that oriented contact is preserved [Blaschke 1929]. Consequently, Laguerre transformations map $S^{*}$-nets to $S^{*}$-nets which means Laguerre invariance:

\section{Lemma 2.18. Discrete $S^{*}$-nets are Laguerre invariant.}

This lemma comes in complete symmetry to the S-net case which says that discrete S-nets are Möbius invariant (see Lemma 2.15).

Note that $S^{*}$-nets naturally discretize the offsetting property of smooth S-nets (cf. Sec. 2.1.3) in contrast to discrete S-nets. The deeper geometric reason for that phenomenon is that "offsetting" is a Laguerre transformation but not a Möbius transformation.

In the dual picture (i.e., $S^{*}$-net setting), the osculating circles are replaced by osculating rotational cones or cylinders in tangential contact to three planes. Consider a net of oriented planes as in Def. 2.17. Three successive planes determine a rotational cone. Three successive planes in the other direction determine another rotational cone. In the case of an $\mathrm{S}^{*}$, the two cones envelope a common sphere.

Definition 2.19. At a plane of an $\mathrm{S}^{*}$-net, the (equal) discrete dual curvature radius $\rho_{n}^{*}$ corresponding to the two directions is the radius of their common tangential sphere. The sphere itself is called discrete Mannheim sphere.

The dual of the tangent of the osculating circle of a discrete S-net at a vertex is the ruling of the rotational cone in tangential contact with three successive planes. Let us denote by $p$ the contact point of the central plane which itself has a normal vector $n$. And let further $q_{1}$ and $q_{2}$ be arbitrary points (i.e., not necessarily

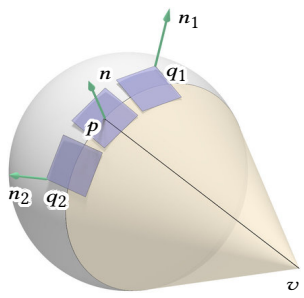
the contact points) on the neighboring planes which have normal vectors $n_{1}$ and $n_{2}$. Then the ruling direction from $p$ to the cone vertex $v$ is parallel to (for a proof of this formula see Appendix A)

$$
\left\langle q_{1}-p, n_{1}\right\rangle n \times n_{2}+\left\langle q_{2}-p, n_{2}\right\rangle n_{1} \times n .
$$

For some applications like flank milling (Sec. 3.2.3) or the construction of certain Weingarten surfaces (Sec. 3.5) we will make use of the specific properties of $S^{*}$-nets. Particularly beneficial are $S^{*}$-nets for flank milling (the milling tool is a surface of revolution acting as milling engagement surface) where the tangent planes naturally envelope the milling tool. For the details on the implementation, the variables, constraints and the algorithm see Section 4.

\subsection{Curvature Theory for S-Nets}

Discrete S-nets discretize a curve network of parameter lines symmetric to principal directions. The discrete parameter lines of S-nets come naturally with a discrete normal curvature (see Def. 2.16). Even more, since every discrete S-net comes with a normal vector field or Gauss map (Def. 2.13), it gives rise to a discrete curvature theory (Section 2.4.2). By comparing specific edge lengths and angles we can further define sensible principal curvatures. To get the setting right it is advisable to look at the smooth case first. 
2.4.1 Smooth principal curvatures. To compute principal curvatures in the setting of S-nets we compare angles between the parameter lines of the S-net and its Gauss map. The goal of this section is to derive formulas for smooth principal curvatures.

We start by expressing the principal directions in terms of tangent vectors of the S-net. Since we are only interested in local curvature properties we do not restrict the following maps to any domain. Let $f: \mathbb{R}^{2} \rightarrow \mathbb{R}^{3}$ be a smooth S-net and let $e_{1}, e_{2}$ denote the principal directions (with $\left\|e_{i}\right\|=1$ ). Let further $n: \mathbb{R}^{2} \rightarrow S^{2} \subset \mathbb{R}^{3}$ denote the Gauss map, i.e., $n=e_{1} \times e_{2}$. Thus the tangent vectors to the parameter lines are $t_{1}:=\frac{f_{u}}{\left\|f_{u}\right\|}=a e_{1}+b e_{2}$ and $t_{2}:=\frac{f_{v}}{\left\|f_{v}\right\|}=a e_{1}-b e_{2}$ for some $a, b \in \mathbb{R}$ with $a^{2}+b^{2}=1$. Then, the tangent vectors of the Gauss map are $v_{1}:=-\kappa_{1} a e_{1}-\kappa_{2} b e_{2}$ and $\nu_{2}:=-\kappa_{1} a e_{1}+\kappa_{2} b e_{2}$ (which are not unit length vectors). Let us define four vectors $\eta_{1}, \eta_{2}, \xi_{1}, \xi_{2}$ parallel to the principal directions (see Figure 10 right):

$$
\eta_{1}:=t_{1}+t_{2}=2 a e_{1}, \quad \eta_{2}:=t_{1}-t_{2}=2 b e_{2},
$$

and

$$
\xi_{1}:=\frac{v_{1}}{\left\|v_{1}\right\|}+\frac{v_{2}}{\left\|v_{2}\right\|}=\frac{-2 \kappa_{1} a e_{1}}{\sqrt{\kappa_{1}^{2} a^{2}+\kappa_{2}^{2} b^{2}}}, \quad \xi_{2}:=\frac{v_{1}}{\left\|v_{1}\right\|}-\frac{v_{2}}{\left\|v_{2}\right\|}=\frac{-2 \kappa_{2} b e_{2}}{\sqrt{\kappa_{1}^{2} a^{2}+\kappa_{2}^{2} b^{2}}} .
$$

Note that the two vectors $\eta_{i}$ and $\xi_{i}$ are parallel. The following determinants indicate a signed ratio of the lengths of orthogonal vectors (oriented by the normal vector)

$$
d_{n}:=\operatorname{det}\left(\frac{\xi_{1}}{\left\|\xi_{1}\right\|^{2}}, \xi_{2}, n\right)=\frac{b \kappa_{2}}{a \kappa_{1}} \text { and } d_{f}:=\operatorname{det}\left(\frac{\eta_{1}}{\left\|\eta_{1}\right\|^{2}}, \eta_{2}, n\right)=\frac{b}{a} \text {, }
$$

and their ratio equals the ratio of the principal curvatures

$$
\frac{d_{f}}{d_{n}}=\frac{\kappa_{1}}{\kappa_{2}} \text {. }
$$

Consequently, we know the ratio between the principal curvatures, but not their magnitude. So we additionally study the angle between the tangent vectors and apply Euler's formula. We denote the cosine of the intersection angle between the parameter curves by $s:=$ $\cos \angle\left(t_{1}, t_{2}\right)=\left\langle t_{1}, t_{2}\right\rangle$. By setting $\varphi=\angle\left(t_{1}, e_{1}\right)$ we can write

$$
s=\cos (2 \varphi)=2 \cos ^{2}(\varphi)-1=1-2 \sin ^{2}(\varphi),
$$

and therefore $\cos ^{2} \varphi=\frac{1+s}{2}$ and $\sin ^{2} \varphi=\frac{1-s}{2}$. Consequently, Euler's formula reads (see Eqn. (2))

$$
\kappa_{n}=\kappa_{1} \cos ^{2} \varphi+\kappa_{2} \sin ^{2} \varphi=\kappa_{1} \frac{1+s}{2}+\kappa_{2} \frac{1-s}{2} .
$$

Together with Equation (6) we obtain

$$
2 \kappa_{n}=\kappa_{2}\left(\frac{d_{f}}{d_{n}}(1+s)+(1-s)\right) .
$$

Finally, we arrive at expressions of the principal curvatures in terms of the radius of the Meusnier sphere and the aforementioned ratios $d_{f}$ and $d_{n}$ :

$$
\kappa_{1}=\frac{2 \kappa_{n} d_{f}}{d_{f}(1+s)+d_{n}(1-s)} \quad \text { and } \quad \kappa_{2}=\frac{2 \kappa_{n} d_{n}}{d_{f}(1+s)+d_{n}(1-s)} .
$$

2.4.2 Discrete principal curvatures. To obtain formulas for discrete principal curvatures in the setting of S-nets we relate analogous notions between the discrete and smooth setting. Consequently, we will construct natural analogs of the notions needed in Sec. 2.4.1.

Let us consider a vertex $\operatorname{star} v, v_{1}, \ldots, v_{4}$ with central vertex $v$. Further, denote the vertex normal at $v$ by $n$ and the four surrounding normals by $n_{1}, \ldots, n_{4}$ at $v_{1}, \ldots, v_{4}$ (each of them is normal to the respective Meusnier sphere.)

The mesh consisting of all vertex normals $n$ itself is an S-net (which is the discrete Gauss map); it lies entirely on the unit sphere.

Our discrete unit tangent vectors at a vertex $v$ are denoted by $t_{1}, t_{2}$. Similarly, we can define unit tangent vectors $v_{1}, v_{2}$ at vertex $n$ of the Gauss map (as tangent vectors to the osculating circles). In analogy to the ingredients for our smooth expressions of the principal curvatures let us denote

$$
\eta_{1}:=t_{1}+t_{2}, \quad \eta_{2}:=t_{1}-t_{2}, \quad \xi_{1}:=v_{1}+v_{2}, \quad \xi_{2}:=v_{1}-v_{2},
$$

and further

$$
d_{n}:=\operatorname{det}\left(\frac{\xi_{1}}{\left\|\xi_{1}\right\|^{2}}, \xi_{2}, n\right) \quad \text { and } \quad d_{v}:=\operatorname{det}\left(\frac{\eta_{1}}{\left\|\eta_{1}\right\|^{2}}, \eta_{2}, n\right),
$$

and the cosine of the angle between the discrete parameter lines by

$$
s:=\cos \angle\left(t_{1}, t_{2}\right) .
$$

Discrete orthogonal S-nets are therefore characterized by $s=0$ or equivalently $d_{v}=1$. The discrete normal curvature $\kappa_{n}$ is defined to be the inverse of the radius of the Meusnier sphere (cf. Def. 2.16). Then our discrete principal curvatures can be defined in analogy to the smooth setting as

$$
\kappa_{1}=\frac{2 \kappa_{n} d_{v}}{d_{v}(1+s)+d_{n}(1-s)} \text { and } \kappa_{2}=\frac{2 \kappa_{n} d_{n}}{d_{v}(1+s)+d_{n}(1-s)} .
$$

Note, that this novel expressions for the discrete principal curvatures are specifically created for "proper" S-nets, i.e., with vertex spheres which do not degenerate to planes. These formulas do not apply to A-nets (i.e., asymptotic nets) where the normal curvature $\kappa_{n}$ vanishes. In that case we would end up with indefinite expressions of the form $0 / 0$.

2.4.3 Orthogonal $S$ - and $S^{*}$-nets. Orthogonal S-nets follow the bisecting directions of the principal curvature directions; these are also the directions of maximal geodesic torsion. Euler's formula (2) implies for smooth orthogonal S-nets, $\kappa_{n}=\kappa_{1} \cos ^{2} \frac{\pi}{4}+\kappa_{2} \sin ^{2} \frac{\pi}{4}=$ $\frac{1}{2}\left(\kappa_{1}+\kappa_{2}\right)=H$, where $H$ is the mean curvature. This property also holds in our discrete setting.

Lemma 2.20. The discrete mean curvature $H_{v}$, i.e., the arithmetic mean of the discrete principal curvatures, at a vertex $v$ of an orthogonal discrete $S$-net is precisely the discrete normal curvature.

Proof. In an orthogonal discrete S-net the cosine of the intersection angle (8) of the discrete parameter curves vanishes, i.e., $s=0$. Thus the arithmetic mean of the discrete principal curvatures (cf. Eqn. (9)) computes to

$$
H_{v}=\frac{\kappa_{1}+\kappa_{2}}{2}=\frac{\kappa_{n} d_{v}}{d_{v}+d_{n}}+\frac{\kappa_{n} d_{n}}{d_{v}+d_{n}}=\kappa_{n},
$$

which implies that the discrete mean curvature equals the discrete normal curvature in these directions.

Orthogonality of $\mathrm{S}^{*}$-nets is measured between the tangential rulings in the tangent plane which is in general different from the angle between the contact curves. In our discrete setting the ruling directions are given by Equation (5). 


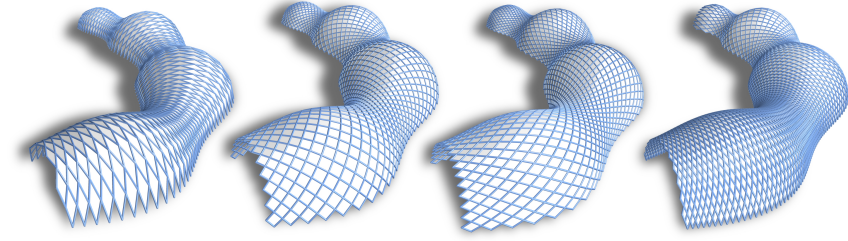

Fig. 11. S-nets with different angles covering the same reference surface. The three nets on the left have constant intersection angles $\pi / 6, \pi / 2$, and $2 \pi / 3$. The intersection angles on the S-net on the right vary strongly over the progression of the mesh.

The involved spheres have a very nice interpretation in terms of discrete differential geometry in sphere geometries (a detailed treatment and comparison to existing discrete curvature notions goes far beyond the scope of the present paper). The Meusnier spheres in orthogonal S-nets have radii $1 / H$ and correspond to the so called central spheres of Möbius geometry whereas the Mannheim spheres in orthogonal $S^{*}$-nets have radii $\left(\kappa_{1}^{-1}+\kappa_{2}^{-1}\right) / 2$ and correspond to the so called mid-spheres of Laguerre geometry [Blaschke 1929, p. 298].

\section{APPLICATIONS}

We model discrete ps nets as quad-dominant meshes $\mathcal{M}(V, E, F)$. Our algorithm solves three basic problems:

(A) approximate a given surface $\mathcal{S}$ with an $\mathrm{S}$ - or $\mathrm{S}^{*}$-net $\mathcal{M}$,

(B) manipulate an existing $\mathrm{S}$ - or $\mathrm{S}^{*}$-net $\mathcal{M}$, and

(C) find vertex positions $V$ for given combinatorics $(E, F)$, such that the resulting net $\mathcal{M}(V, E, F)$ is close to an $\mathrm{S}$ - or $\mathrm{S}^{*}$-net, subject to additional desired properties (Table 2).

The flow of a surface/mesh through our algorithm is shown in Figure 12. Computation of an initial mesh $\mathcal{M}$ from a reference surface $\mathcal{S}$ is described in Section 4.3. When following a path in the pipeline marked with (I), initial values for auxiliary variables (Section 4.2) needed during optimization (Section 4.1) are computed After reaching a result, the user can interact with the mesh by dragging handle vertices (arrow marked III).

In the remainder of this section we present different approximation and modeling tasks that can be performed with our algorithm. We start with the most basic task of approximating a given reference surface with S- or $\mathrm{S}^{*}$-nets (3.1) and show how their properties can be exploited in $\mathrm{CNC}$ milling (3.2). Section 3.4 presents a discrete model

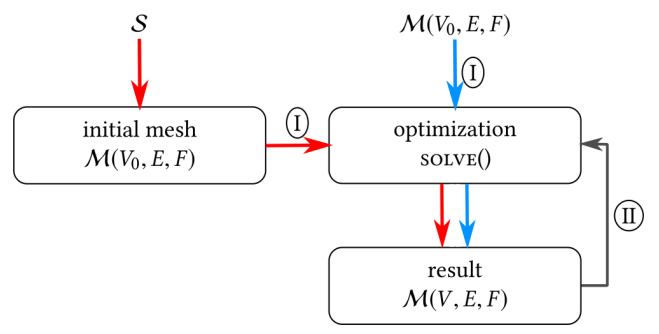

Fig. 12. Computation either starts from a reference surface $\mathcal{S}$ (red path) or mesh $\mathcal{M}$ (blue path). In the first case an initial mesh $\mathcal{M}$ is extracted from $\mathcal{S}$ The user may interact with $\mathcal{M}$, this triggers a new round of optimization.
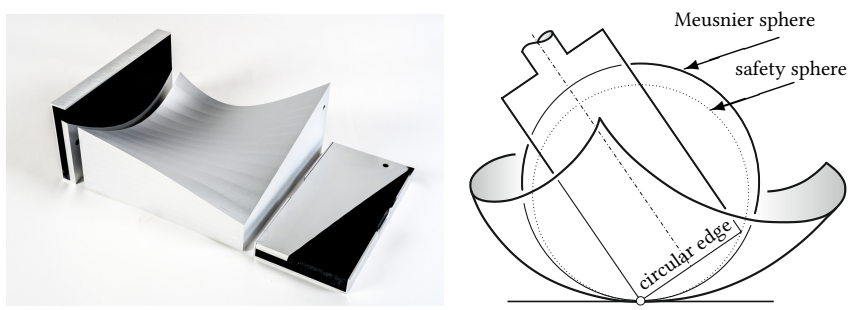

Fig. 13. Left: Milling experiment (cf. Fig. 14 and Sec. 3.2.2). Right: Cylindrical cutter with its circular edge on the savety sphere which is slightly smaller than the Meusnier sphere.

of CMC surfaces represented as S-nets. The remaining subsections cover applications in architecture and design.

\subsection{Discrete S-nets}

It is a surprising fact that $\mathrm{S}$ - and $\mathrm{S}^{*}$-nets bear upon several different applications in so different scales such as architectural facades but also milling small-scale work pieces. In most of our applications the S-net itself is an important part of the design, but for example in the construction of Weingarten surfaces for paneling the shape of the surface that we generate with our ps mesh methods is the output and could be equipped with a different panel layout for its final (generically architectural) application. A compact overview of different surface types together with their properties can be found in Table 2 on page 14.

3.1.1 S-net remeshing. Remeshing given meshes to obtain desired properties is of significant importance in geometry processing. One such application is to generate an S-net over a given surface. That surface shall be given in such a way that we can compute/estimate curvatures and in particular principal directions, for example, as a dense enough triangle mesh or a NURBS surface. Since our approach is optimization based, proper initialization is of prime importance, see Section 4.3. S-net remeshings of typical surfaces appearing in computer graphics are shown in Fig. 3.

Another way of thinking of remeshing in our setting is as generating different S-nets on the same reference surface, i.e., S-nets with different (prescribable) angles. Figure 11 shows three different constant-angle S-nets approximating the same surface as well as one S-net without any angle constraints.

\subsection{Curvature adaptive CNC milling}

CNC machining of freeform surfaces has been the topic of a vast amount of research; see e.g. the survey articles [Harik et al. 2013; Lasemi et al. 2010]. We do not offer a completely new CNC machining solution, but show how ps meshes and in particular Meusnier and Mannheim spheres associated with them provide essential information for curvature adaptive $\mathrm{CNC}$ machining. We are interested in the so-called finishing phase where one wants to achieve a smooth surface. There, it is a big advantage if the cutting tool and its motion are well adapted to the curvature of the surface [Lin et al. 2009]. A study of the relevant literature shows that some contributions result in undercutting, and others do not effectively exploit the available concepts from differential geometry. 
3.2.1 Circumference milling with cylindrical tools. We are cutting with circular edges of a rotational cylindrical tool. For curvature adaptive milling we require that the circular edge is always slightly smaller than an osculating circle of the surface. Recall that osculation (2nd order contact) implies in general a local side change, i.e., local undercutting. Hence, the best practically useful cutting circle will be slightly smaller than the osculating circle. Getting this right is part of the collision test between tool and target surface, which one has to do anyway and for which a variety of algorithms are available.

We achieve curvature adaptation by computing tool positions in such a way that the circular base always lies on a sphere (safety sphere) which has a slightly smaller radius than the Meusnier sphere (see Figure 13 right). This sphere gives us direct access to the tool position. If the sphere radii are constant then also the tool angle is constant. A limitation is that one can only mill from the side where the Meusnier spheres lie. However, such a restriction is present for any type of curvature adapted CNC machining.

To test our approach we milled two surfaces (see Figure 14) with our curvature adaptive method and also one of them with conventional methods, i.e., by using a standard CAM software (Sec. 3.2.2). For generating our tool paths, we position the tool such that the base circle is almost osculating one family of parameter lines of our S-net while moving along the other family. Selecting a good tool size comes from the knowledge of Meusnier sphere radii and constraints on the tool angle as well as the collision and accessibility test. Note that the S-net contains all information which is necessary for this tool path planning approach.

The presented method can easily be extended to toroidal tools where the base circle is rounded off. These tools are outer offsets of cylindrical tools at some distance $r$. Offsetting the target surface by $r$ yields the situation we described here.

3.2.2 Milling experiment. The test pieces are machined from EN AW-50, a medium strength aluminum alloy, on a 5-axis vertical machining center with a rotary tilting table using an indexable $90^{\circ}$ face mill with 50mm diameter and five SOMT 09T304 FR inserts (cutting parameters $\mathrm{vc}=80 \mathrm{~m} / \mathrm{min}, \mathrm{fz}=40 \mu \mathrm{m}$ ). Our tool path runs the cutter at the front only.

The reference finish pass is a standard 5-axis operation with the cutter oriented normal to the drive surface and pulled back in axis direction to avoid gouging when necessary, the tool is ran at the radius. The tool path is generated using the 5-axis multi-surface machining operation in SprutCAM 12. To avoid confusing specular highlights, the aluminum surfaces were lightly etched in sodium hydroxid solution to create a matte surface finish.

3.2.3 Flank milling with conical tools. In flank milling we are cutting with a surface of revolution acting as milling engagement surface. We restrict ourselves to cones of revolution which is dual (in our sense) to cutting with circles (as in Sec. 3.2.1). For curvature adaptive milling we require that the cone is always inside an osculating cone of the surface. It is tangentially circumscribed to a safety sphere, which is slightly smaller than the Mannheim sphere corresponding to the ruling direction. The preferred net type is therefore an $S^{*}$ net, as it determines naturally possible milling tool positions. In Figure 15 we show computed tool paths for the underlying $S^{*}$-net.

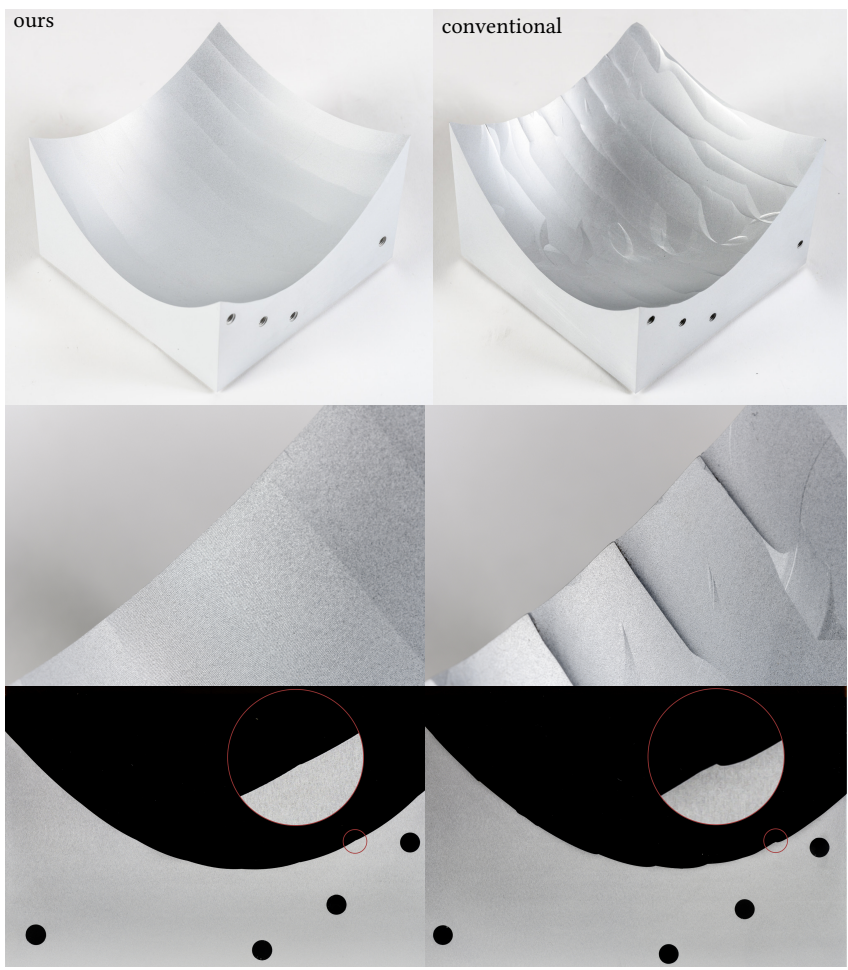

Fig. 14. Comparison of our curvature adaptive milling results (left column) with a conventional method (right column, cf. also Sec. 3.2.1). Our method (see Sec. 3.2.2) results in a much smoother surface as confirmed by the zooming (second row) and the planar section transversally to the tool path motion (third row). Aside: Visualization of our computed tool paths.

The adaption of the cylindrical milling tool $(55 \mathrm{~mm}$ diameter compared to a $15 \mathrm{~cm} \times 15 \mathrm{~cm}$ work piece) to the curvature of the surface is so strong that only 9 sweep paths across the surface where necessary to achieve as little scallop heights as visible in the planar section.

3.2.4 S-nets for milling. S-nets are very suitable as a basis for $\mathrm{CNC}$ tool path planning if the intersection angle is not too far away from 90 degrees, so that one gets sufficiently wide machined strips and high efficiency. Moreover, it is an advantage if the sphere radii do not vary too much, since then one can find a sufficiently big cutter and also does not have to change the tilt angle too much during the machining operation. These two desirable properties need not be strongly conflicting: The sphere radius for an orthogonal S-net at each point equals the harmonic mean of the principal curvature radii. Thus, there is a high chance of having a lower variation in the sphere radii of the S-net than in the principal curvature radii. Another advantage is that S-nets directly provide the integral curves of a field of good machining directions (locally well adapted tool positions) and an overview of the distance variation between adjacent paths. 


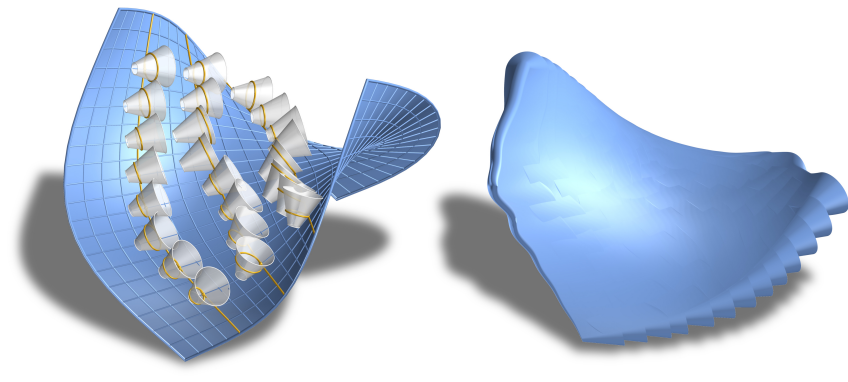

Fig. 15. Curvature adaptive flank milling. In this case the milling tool is a cone of revolution and the milling engagement surface is part of the cone's surface rather than a sharp edge. Dual to path planning in the case of a circular cutting edge, we compute the cones with help of the Mannheim spheres (Sec. 2.1.9) of an $\mathrm{S}^{*}$-net. Our CNC simulation (right) is illustrated by the enveloping surface of the conical tool under the tool path motion that we computed

\subsection{Curved support structures from repetitive elements}

Gridshells are lattice/grid structures which gain strengths by following doubly curved surfaces. Building gridshells by constructing a network of lamellas orthogonal to reference surfaces and by only using repetitive elements was considered in [Schling et al. 2018] They fabricated their lamellas from planar circular strips of constant radius which implies that the curves on the surface along which the lamellas are mounted must follow curves of constant normal curvature. This follows from the fact that the normal curvature of a curve on a surface is preserved in the development of such orthogonal strips [Blaschke and Leichtweiß 1973, p. 83]. A special case is the one of A-nets where the normal curvature is 0 which means asymptotic lines. The corresponding lamelleas develop to elongated rectangles as it is the case in Figure 2. Even more special is the case of A-nets with a constant intersection angle of parameter lines. This class of surfaces and their discretizations which then must have a constant negative ratio of principal curvatures have been studied in [Jimenez et al. 2020].

S-nets (smooth and discrete) with constant radius $r$ Meusnier spheres are nets where both families of parameter lines have constant normal curvature $1 / r$. For reasons of continuity on such surfaces, the Meusnier sphere must always stay on the same side of the surface. Discrete S-nets with constant radius Meusnier spheres are also so-called edge constraint nets in the sense of [Hoffmann et al. 2017], i.e., the sum of neighboring normals is orthogonal to the common edge. This follows immediately from the very symmetric setting since the endpoints of the common edge lie on the intersection circle of congruent spheres and the normals pass through the sphere centers. Another very interesting fact is that the net formed by the Meusnier sphere centers of such a net is also an S-net with constant radius $r$ Meusnier spheres.

3.3.1 Lamellas for curved support. Let us compute a developable lamella sitting orthogonal on a smooth surface along an arbitrary given curve $\gamma$. What we need are the ruling directions $v$ of that developable surface. The tangent vector $t=\dot{\gamma} /\|\dot{\gamma}\|$, the surface normal vector $n$ and the tangent normal $g=n \times t$ form the so called

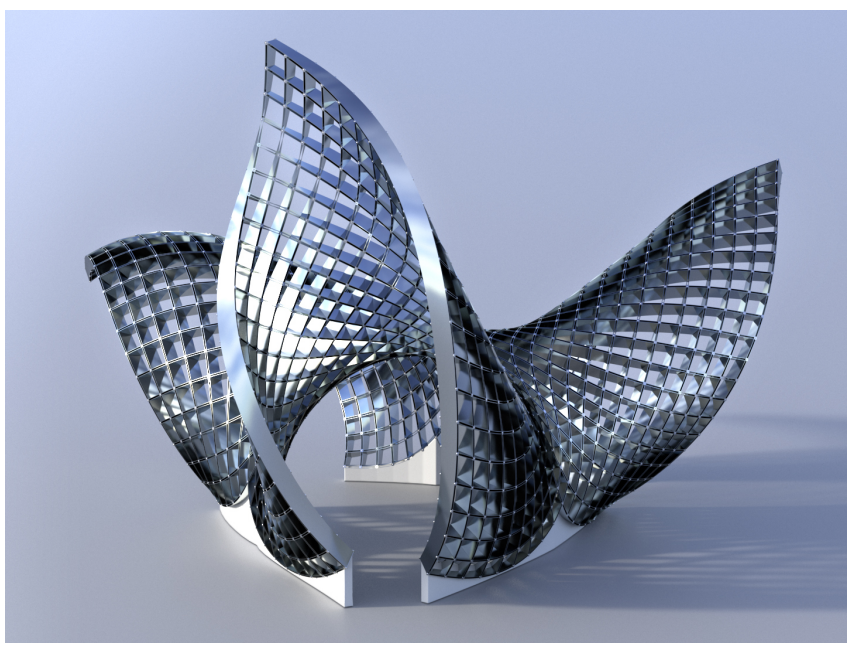

Fig. 16. Rendering of a pavilion, inspired by the work of E. Schling (Fig. 2) The underlying surface is a discrete $S$-net with constant radius Meusnier spheres, resulting in lamellas sitting orthogonal on the surface and which can be unfolded into parts of circular annuli of constant radius.

orthogonal Darboux frame. Then the ruling vector $v$ is computed by $v=g \times \dot{g}=g \times\left(\tau_{g} n-\kappa_{g} n\right)=\tau_{g} t+\kappa_{g} n$, where $\tau_{g}$ is the geodesic torsion and $\kappa_{g}$ the geodesic curvature (see, e.g., [do Carmo 1976, p. 248]).

Figure 16 illustrates a structure with curved developable lamellas orthogonally attached to the underlying surface. This surface is a discrete S-net with constant radius Meusnier spheres implying that the lamellas unfold to the plane to congruent circular strips (annuli). This is a big advantage in fabrication as the preparation of each individual unfolded strips is the same (except for the slits). The paper model in Figure 17 was solely built from circular annuli cut out of paper. The underlying surface is again an S-net with constant Meusnier sphere radii.

3.3.2 Constant ratio of principal curvature Weingarten surfaces. A special case of S-nets with constant sphere radii is the class of $A-$ nets where the radii are $\infty$, i.e., planar vertex stars. The lamellas along such surfaces unfold to planar (elongated) rectangles. If we

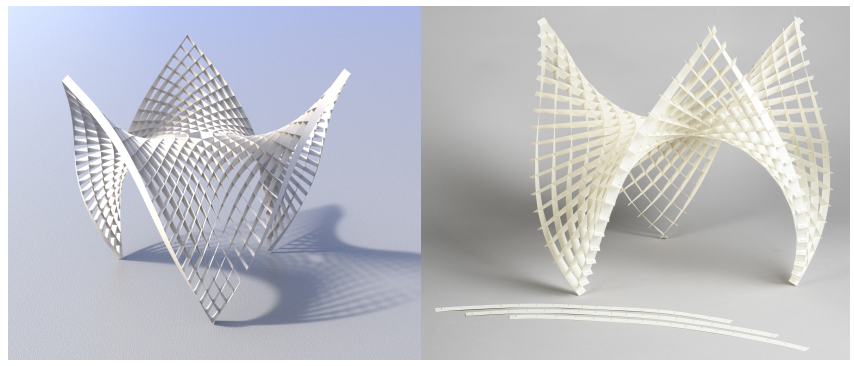

Fig. 17. Left: Rendering of a paper stripmodel with strips orthogonally attached to the reference surface which is an S-net with constant Meusnier sphere radii. Right: Photo of the real built stripmodel together with unfolded strips. All strips are congruent circular annuli of the same radius. 

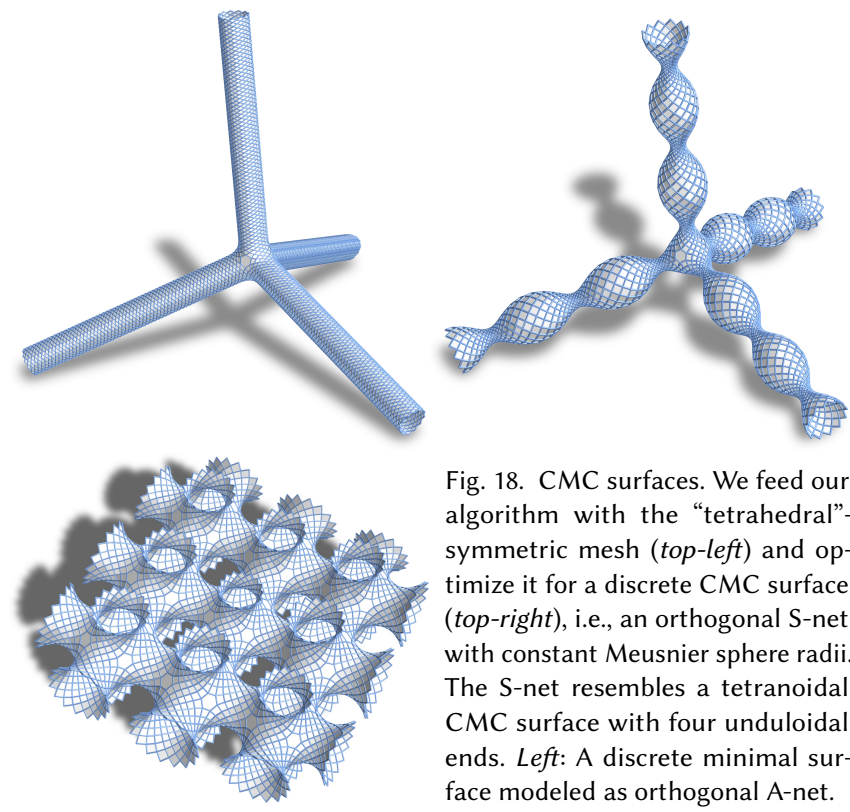

Fig. 18. CMC surfaces. We feed our algorithm with the "tetrahedral"symmetric mesh (top-left) and optimize it for a discrete CMC surface (top-right), i.e., an orthogonal S-net with constant Meusnier sphere radii. The S-net resembles a tetranoidal CMC surface with four unduloidal ends. Left: A discrete minimal surface modeled as orthogonal A-net.

have additionally constant intersection angles we arrive at discrete Weingarten surfaces with $\kappa_{1} / \kappa_{2}=c<0$.

\subsection{CMC surfaces}

There are several computational methods to construct constant mean curvature (CMC) surfaces with the most recent one [Pan et al. 2012] about robust computation methods of discrete CMC surfaces with fixed given or free boundaries. While their method performs computations on a type of Voronoi tesselation and naturally neglects the mesh layout as part of the design, ours is inherently connected to it. We design discrete CMC surfaces from the given combinatorics of the principal curvature net (in fact, its diagonal net).

Orthogonal S-nets where both parameter curves have constant normal curvature $\kappa_{n}=c$ are CMC surfaces since $H=\kappa_{n}=c$ which follows immediately from (10). Consequently, discrete orthogonal $\mathrm{S}$-nets with constant radius Meusnier spheres are CMC surfaces.

In Figure 18 we show a discrete CMC surface with four (unduloidal) ends together with the mesh for the initialization of our algorithm. We optimize for an S-net, orthogonality, and equal Meusnier sphere radii.

Minimal surfaces are surfaces with vanishing mean curvature. Consequently, in our discrete setting, discrete minimal surfaces are orthogonal A-nets (see Figure 18 (bottom-left) for such a net) or S-nets with vanishing discrete mean curvature, i.e., with $d_{n}=-d_{f}$ (see Eqn. (7)) since Equation (9) immediately implies $\kappa_{1}=-\kappa_{2}$ and therefore $H=0$.

\subsection{Mold reduction - Weingarten surfaces}

Weingarten surfaces are surfaces whose principal curvatures $\kappa_{1}, \kappa_{2}$ satisfy an equation $F\left(\kappa_{1}, \kappa_{2}\right)=0$. Typical examples include minimal surfaces $\left(F=\kappa_{1}+\kappa_{2}\right)$, CMC surfaces $\left(F=\kappa_{1}+\kappa_{2}-c\right)$, constant Gaussian curvature surfaces $\left(F=\kappa_{1} \kappa_{2}-c\right)$, surfaces of revolution,
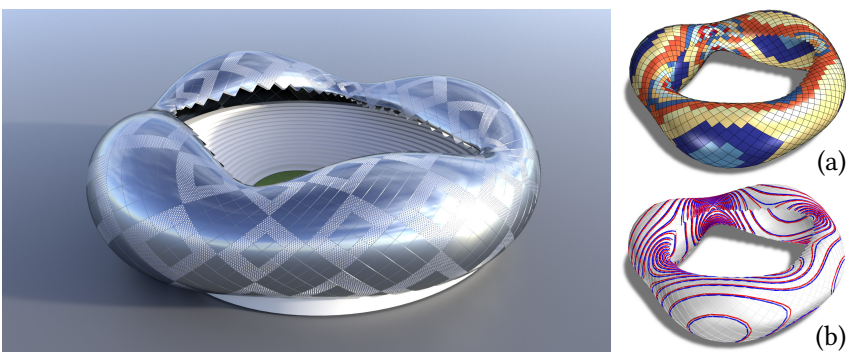

Fig. 19. Rendering of the facade of a stadium. The surface is a Weingarten surface modeled as S-net with constant intersection angle and constant Meusnier sphere radii. The number of molds used for the fabrication of its doubly curved panels reduces approximately to the square root of the number of panels as identically curved panels lie along curves of constant curvature illustrated by isolines (b). Panels with identical molds are clustered together and highlighted by the same color (a).

etc. The significant advantage of such surfaces for architectural panelizations is that it is covered by a one-parameter family of isolines along which both principal curvatures are constant. Therefore along these isolines we can place panels fabricated from the same mold since the curvature behavior is everywhere the same. Compared to non-Weingarten surfaces where the number of necessary molds can be expected to be approximately the number of panels $N$ in the case of Weingarten surfaces they reduce to a magnitude of $\sqrt{N}$.

3.5.1 Affine linear Weingarten surfaces. Euler's formula for isogonal S-nets (i.e., S-nets with constant intersection angle between their parameter lines) with a constant sphere radius reads $a \kappa_{1}+b \kappa_{2}=c$ for some fixed $a, b, c \in \mathbb{R}$. It is therefore a discrete Weingarten surface with an affine linear relation in the principal curvatures. An illustration of such a panelization, the pattern of the isolines and an architectural rendering is shown in Figures 1 and 19. The panels on that surface have been generated with the methods in [Eigensatz et al. 2010] which generate in that case paraboloids that even out smoothness, kink angles and gaps between neighboring panels within real-world tolerances.

3.5.2 Constant harmonic mean curvature surfaces. Blaschke's formula for (smooth) orthogonal $\mathrm{S}^{*}$-nets reads

$$
\rho_{n}^{*}=\frac{1}{\kappa_{2}} \cos ^{2} \theta+\frac{1}{\kappa_{1}} \sin ^{2} \theta=\frac{1}{2}\left(\frac{1}{\kappa_{2}}+\frac{1}{\kappa_{1}}\right),
$$

which is the inverse of the harmonic mean of the principal curvatures. Therefore, if a discrete orthogonal $\mathrm{S}^{*}$-net assumes additionally constant radius Mannheim spheres at each plane these nets are discrete constant harmonic mean curvature surfaces (see image aside) and clearly belong to the class of Weingarten surfaces. Multiplying the above equation by $\kappa_{1} \kappa_{2}$ we obtain an equation of type $H=c K$

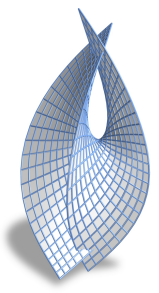
(i.e., according to some authors a linear Weingarten surface). All constant harmonic mean curvature surfaces are offset surfaces of minimal surfaces. 

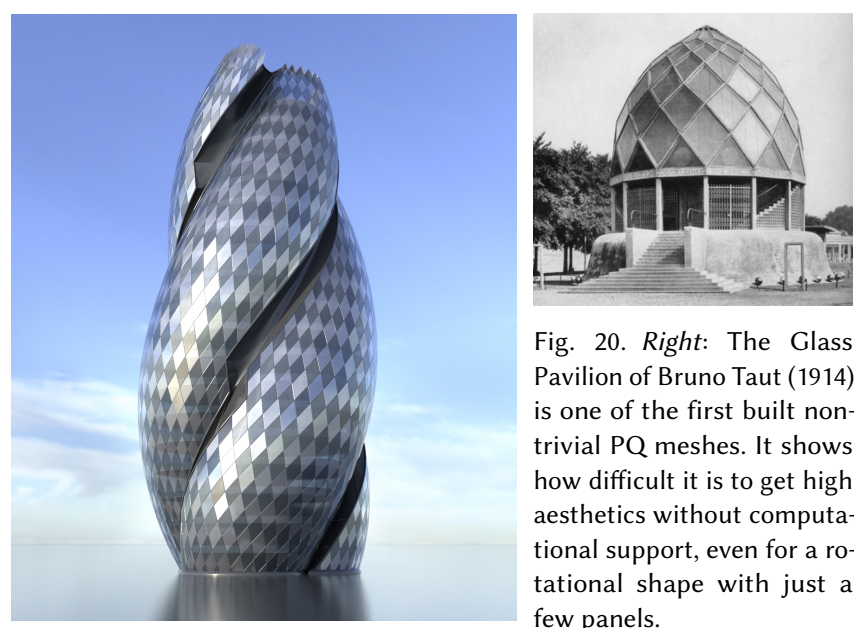

Fig. 20. Right: The Glass Pavilion of Bruno Taut (1914) is one of the first built nontrivial PQ meshes. It shows how difficult it is to get high aesthetics without computational support, even for a rotational shape with just a few panels.

Left: An architectural rendering of a tower consisting of three facade surfaces, each of them with a panelization along the discrete characteristic conjugate net (Sec 3.6). We modeled all faces as planar dimmed glass panels. From the infinite number of conjugate nets (which exist on any surface) this discrete characteristic conjugate net exhibits a more exciting aesthetic impression than a principal net on that surface would do (which would also be conjugate).

\subsection{S-nets with planar faces}

Of great interest in architectural applications and in particular from a manufacturing point of view are nets with planar faces. Planar quad meshes are conjugate nets, i.e., the parameter lines follow conjugate directions. S-nets with planar faces are called characteristic conjugate $n e t s$. In a tangent plane spanned by the principal directions $e_{1}, e_{2}$, the characteristic conjugate directions are given by $t_{1}=\left|\kappa_{1}\right|^{-1 / 2} e_{1}+$ $\left|\kappa_{2}\right|^{-1 / 2} e_{2}$ and $t_{2}=\left|\kappa_{1}\right|^{-1 / 2} e_{1}-\left|\kappa_{2}\right|^{-1 / 2} e_{2}$ (see also Sec 2.1.5 and Figure 5 right). The limitation however of such nets is that they do not exist on negatively curved surfaces. The Swiss Re Tower by $\mathrm{N}$. Foster and K. Shuttleworth is a hybrid version of that idea. It contains several diamond-shaped planar faces which are completed by triangular faces.

Our design of a tower Figure 20 is integrating three facade surfaces, each of them being constructed as a discrete characteristic conjugate net. All faces are modeled as planar glass panels.

\subsection{Circular arc structures}

Another feature of S-nets is the existence of an underlying circular arc structure in the sense of [Bo et al. 2011], where the edges of the net are modeled as circular arcs that touch a common tangent plane at common vertices. S-nets naturally come with such a structure as we can simply intersect neighboring spheres sharing a common edge. By replacing edges with these intersection circles (or better parts of these circles) we obtain a circular arc structure since all arcs emanating from one vertex lie on the same sphere and therefore share a common tangent plane. The appearance of such circle-arcpatterns depends strongly on the variation of the radii of the spheres. A very regular pattern of such an arc structure is shown in Figure 21. We leave further investigations on S-nets with interesting circular arc structures for future research.

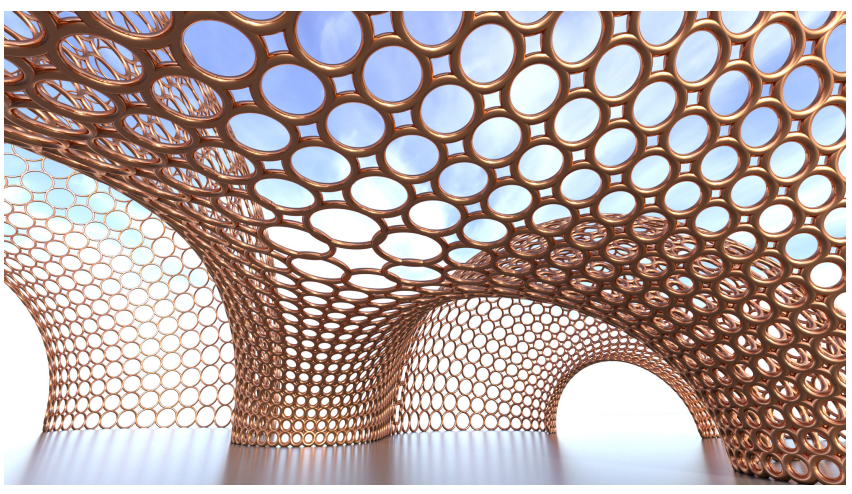

Fig. 21. Circular arc structure. The visual impression of this circular arc structure is the one of a freeform circle packing. However the circles that we see actually consist of four joint circular arcs (right). All circular arcs emanating from one common vertex are in tangential contact with the same tangent plane at that vertex since the circular arcs are parts of the intersection circles of adjacent spheres of an S-net.

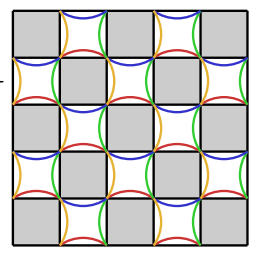

\section{ALGORITHM}

S- or $\mathrm{S}^{*}$-nets are characterized by constraints (Def. 2.14 and 2.17). We encode such constraints as functions $\varphi_{i}: \mathbb{R}^{N} \rightarrow \mathbb{R}$ and express their validity as $\varphi_{i}(x)=0$. The vector $x$ of variables contains vertex coordinates as well as auxiliary variables (see Section 4.2 ). Hence, numeric computation of S- and S*-nets (more precisely, finding the vertex coordinates for given combinatorics) amounts to finding a solution of a system of non-linear equations $\varphi_{i}(x)=0, i=1, \ldots, m$. In the following sections we present the numerical procedure that we use to find such solutions, describe initialization, and give a complete list of used constraint functions and auxiliary variables.

\subsection{Computing constrained meshes}

For concise notation let $F: \mathbb{R}^{N} \rightarrow \mathbb{R}^{m}$,

$$
F(x):=\left(\begin{array}{c}
\varphi_{1}(x) \\
\vdots \\
\varphi_{m}(x)
\end{array}\right), \quad F^{\prime}(x):=\left(\begin{array}{c}
\nabla \varphi_{1}(x)^{T} \\
\vdots \\
\nabla \varphi_{m}(x)^{T}
\end{array}\right) .
$$

In our setting the system $F(x)=0$ is underconstrained, otherwise there would be no d.o.f. left for design. Hence, Newton's method cannot be directly applied. To circumvent this problem we apply regularized least squares to solve $F^{\prime}(x) h=-F(x)$, i.e., we minimize

$$
\left\|F^{\prime}(x) h+F(x)\right\|^{2}+\varepsilon\|h\|^{2} .
$$

Solving the corresponding weighted normal equations in each iteration leads to Algorithm 1 which forms the basis of our optimization.

As shown in [Tang et al. 2014] this approach is especially efficient when the functions $\varphi_{i}$ are polynomials of degree two (or less) and 'sparse' in the vector $x$. By this we mean that a function $\varphi_{i}$ only depends on very few entries of $x$, typically the coordinates and associated auxiliary variables of a vertex and those of its neighboring vertices. This yields sparse, positive definite matrices $A^{T} A+\varepsilon I$ and facilitates the use of efficient numerical methods, like sparse 
Table 1. Variables per vertex, edge, or face. The last column gives the total number of each type. In total we have $28|V|+|E|+3|F|+2$ variables.

\begin{tabular}{|l|l|c|}
\hline description & names & \# \\
\hline \hline vertex positions for each vertex & $v \in \mathbb{R}^{3}$ & $3|V|$ \\
\hline coefficients of sphere equations at each vertex star & $\begin{array}{l}a, b_{1}, b_{2}, \\
b_{3}, c \in \mathbb{R}\end{array}$ & $5|V|$ \\
\hline auxiliary variable guaranteeing positivity of $a$ & $A \in \mathbb{R}$ & $|V|$ \\
\hline auxiliary variable for vertex norm $\|v\|$ & $w \in \mathbb{R}$ & $|V|$ \\
\hline squared edge lengths for each edge & $L \in \mathbb{R}$ & $|E|$ \\
\hline tangent vectors to osculating circles at $v$ & $t_{1}, t_{2} \in \mathbb{R}^{3}$ & $6|V|$ \\
\hline length of tangent vectors at $v$ & $s_{1}, s_{2} \in \mathbb{R}^{3}$ & $2|V|$ \\
\hline unit tangent vectors at $v$ & $e_{1}, e_{2} \in \mathbb{R}^{3}$ & $6|V|$ \\
\hline face normal & $n \in \mathbb{R}^{3}$ & $3|F|$ \\
\hline vertex normal for coherent sphere alignment & $N_{s} \in \mathbb{R}^{3}$ & $3|V|$ \\
\hline auxiliary variable for coherent sphere alignment & $k_{s} \in \mathbb{R}$ & $|V|$ \\
\hline auxiliary variables for equal radii and angles & $r, \theta_{2} \in \mathbb{R}$ & 2 \\
\hline
\end{tabular}

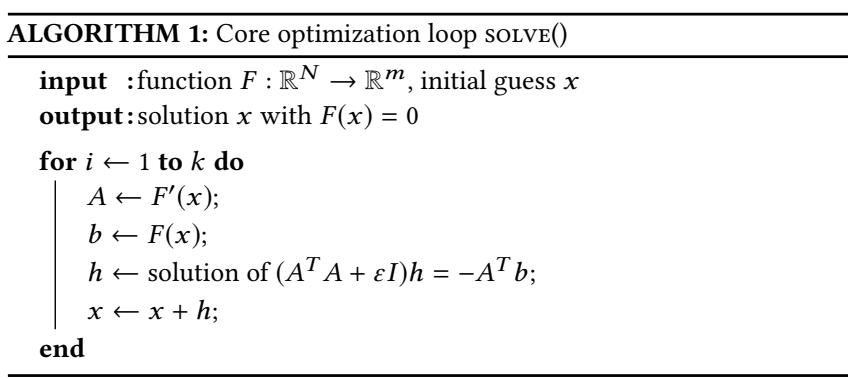

Cholesky factorization to solve the weighted normal equations. As proposed in [Tang et al. 2014] we use values $k \leq 10$ and $\varepsilon=0.001$.

\subsection{Variables and constraints}

We work with quad-dominant meshes $\mathcal{M}(V, E, F)$ with mainly valence 4 vertex stars. Our constraint functions $\varphi_{i}$ are defined locally - involving only a vertex and at most its 1-ring neighborhood. In the generic case of a valence 4 vertex we refer to the 1-ring neighbors of $v$ as $v_{1}, v_{2}, v_{3}, v_{4}$. In addition to vertex coordinates we also use auxiliary variables that are used in the definition and evaluation of constraints. Some of those auxiliary variables only serve the purpose of reducing the degree of a constraint to 2 . Auxiliary variables are either attached to vertices, edges, or faces of $\mathcal{M}$. Table 1 lists all variables and how often they occur.

Our implementation is based on a generic half-edge data structure which makes it easy to augment the definition of vertices, edges, and faces with additional data blocks representing associated auxiliary variables. In the following we present all our constraint functions which, in different combinations (see Table 2), lead to nets with different properties.

- Spherical nodes. A sphere can be represented as the zero set of

$$
f(x)=a x^{2}+\left\langle\left(b_{1}, b_{2}, b_{3}\right), x\right\rangle+c=0,
$$

where the center is $\frac{-1}{2 a}\left(b_{1}, b_{2}, b_{3}\right)$ and the radius equals $\frac{1}{2|a|}\left(b_{1}^{2}+\right.$ $\left.b_{2}^{2}+b_{3}^{2}-4 a c\right)^{1 / 2}$. In that case the coefficients can be normalized s. $t$

$$
\|\nabla f\|^{2}=b_{1}^{2}+b_{2}^{2}+b_{3}^{2}-4 a c=1 .
$$

Table 2. List of different types of S-nets (black) and $\mathrm{S}^{*}$-nets (blue) together

\begin{tabular}{|c|c|c|c|c|c|c|}
\hline 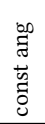 & 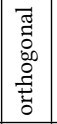 & 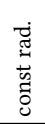 & 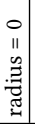 & 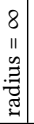 & & name \\
\hline- & - & - & - & - & - & general S-net/S*-net \\
\hline $1 / 1$ & - & - & - & - & - & isogonal S-net/S*-net \\
\hline $1 / 1$ & $1 / 1$ & - & - & - & - & orthogonal S-net/S*-net \\
\hline- & - & $1 / 1$ & - & - & - & $\begin{array}{l}\text { const. normal curvature S-net/const. dual curvature } \\
\text { radius S*-net }\end{array}$ \\
\hline 1 & 1 & $1 / 1$ & - & - & - & CMC S-net/const. harmonic mean curvature surf. \\
\hline- & - & 1 & - & 1 & - & A-net \\
\hline- & - & 1 & 1 & - & - & dual A-net \\
\hline 1 & - & 1 & - & - & - & affine linear Weingarten S-net \\
\hline 1 & 1 & 1 & - & 1 & 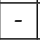 & minimal surface as A-net \\
\hline 1 & - & 1 & - & 1 & - & negative constant ratio of principal curvature A-net \\
\hline- & - & - & - & - & 1 & characteristic conjugate S-net \\
\hline 1 & $1 / 1$ & - & - & - & - & mean curvature spheres/mid curvature spheres \\
\hline
\end{tabular}
with their characterizing properties.

Then the radius is $\frac{1}{2|a|}$. Equation (13) represents a plane if and only if $a=0$. Therefore an S-net is a net with

$$
f(v)=0, \quad f\left(v_{i}\right)=0 \quad \text { for } i=1, \ldots, 4,
$$

for each vertex $\operatorname{star} v, v_{1}, \ldots, v_{4}$. Since the expression $a \cdot\|v\|^{2}$ is not quadratic we introduce auxiliary variables $w$ subject to $w-\|v\|^{2}=0$ in order to make $f(v)$ quadratic.

- Guaranteeing positivity of a. To work with $\frac{1}{2 a}$ as radius, we have to guarantee that $a$ is positive, i.e., by an equation of the form $a-A^{2}=0$ at each vertex star of valence four.

- Equal normal curvature. Equality of all sphere radii implies equality of all normal curvatures. If Equation (14) holds then the radius of each sphere is $1 /(2 a)$ and the constraint for globally equal radii $r$ reads $2 a r-1=0$ at each vertex star.

- Tangent vectors to osculating circles. Osculating circles of the parameter lines are circumcircles of $v_{1} v v_{3}$ and $v_{2} v v_{4}$. At vertex $v$, Lemma 2.12 implies for the tangent vectors $\left(v_{1}-v\right) L_{3}-\left(v_{3}-v\right) L_{1}-t_{1}=$ 0 and $\left(v_{2}-v\right) L_{4}-\left(v_{4}-v\right) L_{2}-t_{2}=0$, where $L_{j}$ are the squared edge lengths, i.e., $\left\|v_{j}-v\right\|^{2}-L_{j}=0$.

- Constant angle. We measure the angle between the unit vectors $e_{1}, e_{2}$ to the osculating circles. Consequently, we must relate the above tangent vectors $t_{1}, t_{2}$ with their lengths $s_{1}, s_{2}$ to $e_{1}, e_{2}$ by $t_{1}-s_{1} e_{1}=0$ and $t_{2}-s_{2} e_{2}=0$, together with $\left\|e_{1}\right\|^{2}-1=0$ and $\left\|e_{2}\right\|^{2}-1=0$. Then the angle $\theta$ obeys $\left\langle e_{1}, e_{2}\right\rangle-\cos \theta_{0}=0$.

- Planar faces. To ensure planarity of faces, we introduce a normal vector $n$ and enforce orthogonality by $\left\langle v_{i}-v_{j}, n\right\rangle=0$ and for all edge vectors of the face and $\langle n, n\rangle=1$ such that $n$ does not degenerate.

- Coherent sphere alignment. For some applications we need the spheres to coherently lie on one side of the surface. For that we compare the outward pointing normal $N_{s}$ with the reference normal $N_{r}$ of the mesh by requiring $\left\langle N_{s}, N_{r}\right\rangle=k_{s}^{2}$ and $N_{s}-2 a v-\left(b_{1}, b_{2}, b_{3}\right)=0$. 
- Distance to a reference surface. When approximating a reference surface $\mathcal{S}$, the mesh $\mathcal{M}$ is supposed to 'glide' on $\mathcal{S}$. This behavior is realized by computing footpoints $p_{k} \in \mathcal{S}$ and corresponding normal vectors $n_{k}$ for each vertex $v_{k} \in V$. Closeness to $\mathcal{S}$ is enforced by minimizing point-to-plane distances $\left\langle v_{k}-p_{k}, n_{k}\right\rangle=0$. The $p_{k}$ and $n_{k}$ are updated after each iteration of Alg. 1. Using an efficient data structure (e.g. [Muja and Lowe 2009]) to realize the projection operator $V \rightarrow \mathcal{S}$ greatly improves the performance of our algorithm.

\subsection{Initialization}

4.3.1 Finding the right combinatorics. Edges of an S-net $\mathcal{M}$ around a vertex are symmetric with respect to the principal curvature directions at that vertex. If we are given a reference surface $\mathcal{S}$ to be approximated by an $\mathrm{S}$ -

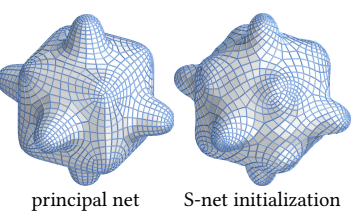
net, our initial guess for $\mathcal{M}$ should be close to such a geometric configuration. So, we first estimate principal curvature directions on $\mathcal{S}$ on a per face basis, cf. [Rusinkiewicz 2004]. The cross field obtained in this way is then rotated by $45^{\circ}$ and integrated, cf. [Bommes et al. 2009]. We use the resulting parametrization of $\mathcal{S}$ to extract our initial guess $\mathcal{M}$, [Ebke et al. 2013]. This procedure generates the vertex set $V$ and combinatorics $(E, F)$ (including automatic placement of singularities) of a quad-dominant mesh $\mathcal{M}$. Note that the combinatorics of $\mathcal{M}$ does not change during the optimization.

One way of making $S^{*}$-nets computationally accessible is by contact elements (i.e., contact point plus normal direction) instead of just a net of planes. The advantage of knowing the contact point where the sphere touches the plane gives direct access to the underlying ps net and makes computations like intersection angle or fairness energy evaluations feasible. As initial mesh for computation of $\mathrm{S}^{*}$-nets we take the geometry of an S-net and approximate the Mannheim spheres by the tangent plane information that we are given in the vertices. Note that in general the Mannheim spheres corresponding to $S^{*}$-nets and the Meusnier spheres corresponding to S-nets can be very different even though the geometry of the nets seen as surfaces look similar.

4.3.2 Singularities and fairness. The constraints listed above do not imply any kind of mesh fairness. To achieve smooth meshes we apply a fairness term with a very small weight, typically in the range $10^{-6}$ to $10^{-3}$. Fairness is most important during early iterations of optimization and may be faded out when approaching a solution, see [Tang et al. 2014]. A mesh generated as described in the previous paragraph has singularities whenever the cross field of principal directions exhibits singularities. We do not apply any constraints except fairness around such vertices. For regular vertices fairness is achieved by minimizing second differences $v_{1}-2 v+v_{3}=0$ and $v_{2}-2 v+v_{4}=0$. For valence $n \neq 4$ vertices we use the umbrella operator $\sum_{i=1}^{n} v_{i}-n v=0$ as fairness term.

4.3.3 Initialization of auxiliary variables. Vertex coordinates $V$ are fixed in this step. Since all auxiliary variables have a geometric meaning we can use standard tools to estimate them, e.g., for a vertex $v$ we get $a, b_{1}, b_{2}, b_{3}, c$ by computing a least squares sphere
Table 3. The computation times collected in the last column refer to seconds per iteration as our implementation is a handle-based editing method.

\begin{tabular}{|c|l|c|c|c|}
\hline Fig & constraints & $|V|$ & $\mid$ var $\mid$ & T/iter \\
\hline 1 & linear Weingarten & 2824 & 79165 & $1.03 \mathrm{~s}$ \\
\hline $3-1$ & S-net & 3408 & 33750 & $0.78 \mathrm{~s}$ \\
\hline $3-2$ & S-net & 4992 & 49920 & $1.11 \mathrm{~s}$ \\
\hline $3-3$ & S-net & 2731 & 26914 & $0.55 \mathrm{~s}$ \\
\hline 15 & S*-net + coh. sphere orient. & 441 & 8476 & $0.10 \mathrm{~s}$ \\
\hline 16 & const. radius spheres & 1205 & 15131 & $0.22 \mathrm{~s}$ \\
\hline 18 -left & A-net + orthogonal & 6096 & 148416 & $2.47 \mathrm{~s}$ \\
\hline 19 & linear Weingarten & 1984 & 56321 & $0.76 \mathrm{~s}$ \\
\hline
\end{tabular}

fit to $v, v_{1}, v_{2}, v_{3}, v_{4}$. Analogously we can initialize face normals. A formula for the computation of tangent vectors to osculating circles is given in Lemma 2.12 .

\subsection{Convergence behavior}

As with any numerical optimization, convergence depends on the initial guess. In our setting there are two aspects to this: initial vertex positions $V$ and mesh combinatorics $(E, F)$. A fixed mesh topology and a chosen set of constraints defines a solution manifold, i.e., an admissible set of vertex coordinates. Initial vertex coordinates $V$ need to be reasonably close to the solution manifold for our optimization to succeed. We discuss the convergence behavior of our method for each basic task.

(A) Our initialization procedure ensures that the initial vertex positions as well as the combinatorics are close to a valid S- or $\mathrm{S}^{*}$ net. We did not experience convergence problems in this setting. The situation is analogous to that of computing a quad mesh with planar faces, i.e., one has to start with a conjugate curve network in order to faithfully approximate a reference surface [Sauer 1970].

(B) Applying interactive changes to vertex coordinates of an Sor $\mathrm{S}^{*}$-net can lead to configurations where the mesh topology can no longer sustain the ps property. Since we treat vertex positions of handle vertices as hard constraints, the optimization cannot fix this and fails.

(C) In contrast to (B) there are no hard constraints on any vertex coordinates. Figure 18 (top row) shows an example of this 'form finding' procedure. If the initial set of vertex coordinates is too far away from the solution manifold the mesh will collapse to a single point. This behavior is due to the fairness energy.

\subsection{Computation times}

As most of our application oriented examples involve interactive design steps, we only provide computation times for each iteration of Algorithm 1. While moving a vertex we run one iteration and after releasing the mouse button five more. The computation times in Table 3 refer to our implementation in Python on a $2.7 \mathrm{GHz}$ Intel ${ }^{\circledR}$ Core $^{\mathrm{TM}}$ i5 CPU with 8GB RAM.

\section{CONCLUSION}

\subsection{Limitations}


On simple surfaces, the increased flexibility of S-nets allows one to design visually pleasing and interesting alternatives to the rather monotonous quad meshes which are aligned with principal directions. However, when a surface possesses details with high curvature variation, Snets may not be the method of choice.

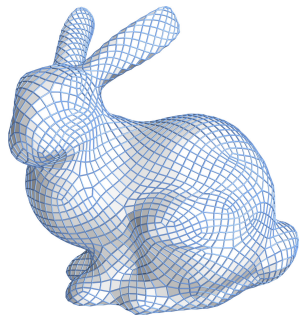
This is seen in the Bunny model aside.

It is far beyond the scope of our paper to provide a complete path planning algorithm for $\mathrm{CNC}$ machining. This requires more studies, but apart from that, there are unavoidable and already mentioned limitations for all types of curvature adapted machining.

\subsection{Conclusion and future research}

We have presented the first in-depth study of quad meshes which are symmetric with respect to principal directions. Depending on the type of sphere geometry which underlies the discretization, we investigated two types of these ps meshes and showed how to compute them with numerical optimization. Moreover, we outlined their potential for various applications in design, architecture and computational fabrication.

We could not elaborate on all aspects in full detail and completeness and thus there is plenty of room for future research.

On the theoretical side, one can unify the two types of meshes within Lie sphere geometry [Blaschke 1929] and obtain quad meshes of oriented spheres in which each sphere and its four connected neighboring spheres are in oriented contact with a sphere. There is also a need for a better understanding of the discrete differential geometry of those special surfaces which can be elegantly represented as ps meshes.

Concerning applications, most of the ones we addressed deserve a closer investigation. This is particularly true for remeshing exploiting the degrees of freedom and guided by the specific application. The latter could be CNC machining, where some of the open issues have been indicated above.

\section{ACKNOWLEDGMENTS}

The authors gratefully acknowledge the support by the SFB-Transregio "Discretization in Geometry and Dynamics" through grant I 2978 of the Austrian Science Fund (FWF) as well as by project F77 (SFB “Advanced Computational Design”, subproject SP6), by project P 29981, and by KAUST grant OSR\#4009. Furthermore, the authors would like to thank the anonymous reviewers for their valuable comments.

\section{REFERENCES}

Wilhelm Blaschke. 1916. Kreis und Kugel. Verlag von Veit und Comp.

Wilhelm Blaschke. 1929. Vorlesungen über Differentialgeometrie und geometrische Grundlagen von Einsteins Relativitätstheorie. Band III. Differentialgeometrie der Kreise und Kugeln. Springer, Berlin.

Wilhelm Blaschke and Kurt Leichtweiß. 1973. Elementare Differentialgeometrie. Springer Berlin-New York. Fünfte vollständig neubearbeitete Auflage von K. Leichtweiß, Die Grundlehren der mathematischen Wissenschaften, Band 1.

Pengbo Bo, Helmut Pottmann, Martin Kilian, Wenping Wang, and Johannes Wallner. 2011. Circular Arc Structures. ACM Trans. Graph. 30, 4 (2011), 101:1-101:11

Alexander Bobenko and Yuri Suris. 2006. Isothermic surfaces in sphere geometries as Moutard nets. Proceedings of The Royal Society A Mathematical Physical and Engineering Sciences 463 (2006), 3171-3193.
Alexander I. Bobenko and Peter Schröder. 2005. Discrete Willmore Flow. In Proc. Eurographics Symposium on Geometry Processing. Eurographics Assoc., 101-110.

Alexander I. Bobenko and Yuri B. Suris. 2008. Discrete differential geometry. Integrable structure. Graduate Studies in Mathematics, Vol. 98. American Mathematical Society, Providence, RI.

David Bommes, Bruno Lévy, Nico Pietroni, Enrico Puppo, Claudio Silva, Marco Tarini, and Denis Zorin. 2013. Quad-Mesh Generation and Processing: A Survey. Computer Graphics Forum 32, 6 (2013), 51-76.

David Bommes, Henrik Zimmer, and Leif Kobbelt. 2009. Mixed-Integer Quadrangulation. ACM Trans. Graph. 28, 3, Article Article 77 (July 2009), 10 pages.

Olga Diamanti, Amir Vaxman, Daniele Panozzo, and Olga Sorkine-Hornung. 2015. Integrable PolyVector Fields. ACM Trans. Graph. 34, 4 (2015), 38:1-38:12.

Manfredo P. do Carmo. 1976. Differential geometry of curves and surfaces. Prentice-Hall, Inc., Englewood Cliffs, N.J. Translated from the Portuguese.

Hans-Christian Ebke, David Bommes, Marcel Campen, and Leif Kobbelt. 2013. QEx: Robust Quad Mesh Extraction. ACM Trans. Graph. 32, 6, Article Article 168 (Nov. 2013), 10 pages.

Michael Eigensatz, Martin Kilian, Alexander Schiftner, Niloy J. Mitra, Helmut Pottmann, and Mark Pauly. 2010. Paneling Architectural Freeform Surfaces. ACM Trans. Graph. 29, 4 (2010), 45:1-45:10.

Sebastian Finsterwalder. 1899. Mechanische Beziehungen bei der Flächendeformation Jahresber. d. Deutschen Math.-Vereinigung 6 (1899), 43-90.

Akash Garg, Andrew O. Sageman-Furnas, Bailin Deng, Yonghao Yue, Eitan Grinspun, Mark Pauly, and Max Wardetzky. 2014. Wire Mesh Design. ACM Trans. Graph. 33, 4 (2014), 66:1-66:12.

Ramy F. Harik, Hu Gong, and Alain Bernard. 2013. 5-axis flank milling: A state-of-theart review. Computer-Aided Design 45, 3 (2013), 796-808.

Tim Hoffmann, Andrew Sageman-Furnas, and Max Wardetzky. 2017. A discrete parametrized surface theory in $\mathbb{R}^{3}$. Intl. Math. Research Notices 14 (2017), 4217-4258.

Wenzel Jakob, Marco Tarini, Daniele Panozzo, and Olga Sorkine-Hornung. 2015. Instant Field-Aligned Meshes. ACM Transactions on Graphics (Proceedings of SIGGRAPH ASIA) 34, 6 (2015).

Michael R. Jimenez, Christian Müller, and Helmut Pottmann. 2020. Discretizations of Surfaces with Constant Ratio of Principal Curvatures. Discrete Comput. Geom. 63, 3 (2020), 670-704.

Mina Konaković, Keenan Crane, Bailin Deng, Sofien Bouaziz, Daniel Piker, and Mark Pauly. 2016. Beyond Developable: Computational Design and Fabrication with Auxetic Materials. ACM Trans. Graph. 35, 4 (2016), 89:1-89:11.

Mina Konaković, Julian Panetta, Keenan Crane, and Mark Pauly. 2018. Rapid Deployment of Curved Surfaces via Programmable Auxetics. ACM Trans. Graph. 37, 4 (2018), 106:1-106:13.

Ali Lasemi, Deyi Xue, and Peihua Gu. 2010. Recent development in CNC machining of freeform surfaces: A state-of-the-art review. Computer-Aided Design, 42,7 (2010), 641-657.

Than Lin, Jae-Woo Lee, and Erik Bohez. 2009. A new accurate curvature matching and optimal tool based five-axis machining algorithm. fournal of Mechanical Science and Technology 23 (10 2009), 2624-2634.

Marius Muja and David Lowe. 2009. Fast Approximate Nearest Neighbors with Automatic Algorithm Configuration. VISAPP 2009 - Proceedings of the 4th International Conference on Computer Vision Theory and Applications 1, 331-340.

Hao Pan, Yi-King Choi, Yang Liu, Wenchao Hu, Qiang Du, Konrad Polthier, Caiming Zhang, and Wenping Wang. 2012. Robust Modeling of Constant Mean Curvature Surfaces. ACM Trans. Graph. 31, 4 (2012), 85:1-85:11.

Davide Pellis, Martin Kilian, Felix Dellinger, Johannes Wallner, and Helmut Pottmann 2019. Visual smoothness of polyhedral surfaces. ACM Trans. Graphics 38, 4 (2019), 260:1-260:11. Proc. SIGGRAPH.

Helmut Pottmann, Michael Eigensatz, Amir Vaxman, and Johannes Wallner. 2015. Architectural Geometry. Computers and Graphics 47 (2015), 145-164.

Michael Rabinovich, Tim Hoffmann, and Olga Sorkine-Hornung. 2018a. Discrete Geodesic Nets for Modeling Developable Surfaces. ACM Trans. Graph. 37, 2 (2018), 16:1-16:17.

Michael Rabinovich, Tim Hoffmann, and Olga Sorkine-Hornung. 2018b. The Shape Space of Discrete Orthogonal Geodesic Nets. ACM Trans. Graph. 37, 6 (2018), 228:1228:17.

Szymon Rusinkiewicz. 2004. Estimating curvatures and their derivatives on triangle meshes. In Proceedings. 2nd International Symposium on 3D Data Processing, Visualization and Transmission, 2004. 3DPVT 2004. 486-493.

Andrew O. Sageman-Furnas, Albert Chern, Mirela Ben-Chen, and Amir Vaxman. 2019 Chebyshev Nets from Commuting PolyVector Fields. ACM Trans. Graph. 38, 6 (2019), 172:1-172:16.

Robert Sauer. 1970. Differenzengeometrie. Springer.

Eike Schling. 2018. Repetitive Structures. Ph.D. Dissertation. TU Munich.

Eike Schling, Martin Kilian, Hui Wang, Denis Schikore, and Helmut Pottmann. 2018. Design and construction of curved support structures with repetitive parameters. In Adv. in Architectural Geometry, Lars Hesselgren et al. (Ed.). Klein Publ. Ltd, 140-165. 
Chengcheng Tang, Xiang Sun, Alexandra Gomes, Johannes Wallner, and Helmut Pottmann. 2014. Form-finding with Polyhedral Meshes Made Simple. ACM Trans. Graph. 33, 4 (2014), 70:1-70:9.

Jean-Marc Thiery, Emilie Guy, and Tamy Boubekeur. 2013. Sphere-Meshes: Shape Approximation using Spherical Quadric Error Metrics. ACM Transaction on Graphics (Proc. SIGGRAPH Asia 2013) 32, 6 (2013), 178:1-178:12.

Jean-Marc Thiery, Émilie Guy, Tamy Boubekeur, and Elmar Eisemann. 2016. Animated Mesh Approximation With Sphere-Meshes. ACM Trans. Graph. 35, 3 (2016), 30:130:13.

Anastasia Tkach, Mark Pauly, and Andrea Tagliasacchi. 2016. Sphere-Meshes for RealTime Hand Modeling and Tracking. ACM Trans. Graph. 35, 6 (2016), 222:1-222:11.

Amir Vaxman, Marcel Campen, Olga Diamanti, David Bommes, Klaus Hildebrandt, Mirela Ben-Chen, and Daniele Panozzo. 2017. Directional Field Synthesis, Design, and Processing. In ACM SIGGRAPH 2017 Courses. Article 12, 30 pages.

Amir Vaxman, Christian Müller, and Ofir Weber. 2015. Conformal Mesh Deformations with Möbius Transformations. ACM Trans. Graph. 33, 4 (2015), 55:1-55:11.

Amir Vaxman, Christian Müller, and Ofir Weber. 2018. Canonical Möbius Subdivision. ACM Trans. Graph. 37, 6 (2018), 227:1-227:15.

Hui Wang, Davide Pellis, Florian Rist, Helmut Pottmann, and Christian Müller. 2019 Discrete geodesic parallel coordinates. ACM Trans. Graph. 38, 6 (2019), 173:1-173:13. Proc. SIGGRAPH Asia.

Walter Wunderlich. 1951. Zur Differenzengeometrie der Flächen konstanter negativer Krümmung. Österreich. Akad. Wiss. Math.-Nat. Kl. S.-B. IIa. 160 (1951), 39-77.

\section{A PROOFS OF THEOREMS}

Proposition 2.11. Let us assume that $f$ is an arbitrary parametrization of a surface and $t=a f_{u}+b f_{v}$ is an arbitrary tangent vector. Then the tangent vector $s=\tilde{a} f_{u}+\tilde{b} f_{v}$ is symmetric to $t$ with respect to the principal directions if and only if

$$
(a, b) M\left(\begin{array}{l}
\tilde{a} \\
\tilde{b}
\end{array}\right):=(a, b)\left(\begin{array}{cc}
2 F e-2 E f & G e-E g \\
G e-E g & 2 G f-2 F g
\end{array}\right)\left(\begin{array}{l}
\tilde{a} \\
\tilde{b}
\end{array}\right)=0
$$

i.e., if $(a, b)$ is orthogonal to $(\tilde{a}, \tilde{b})$ with respect to the $2 \times 2$ matrix $M$.

Proof. Let us first assume that the two tangent vectors $s, t$ are linearly independent. The corresponding directions in the parameter domain $(a, b)$ and $(\tilde{a}, \tilde{b})$ therefore fulfill $\tilde{a} b-a \tilde{b} \neq 0$. Due to Lemma 2.2 the condition for an S-net can be translated into a condition of equal normal curvature, i.e., the two directions in the parameter domain $(a, b)$ and $(\tilde{a}, \tilde{b})$ correspond to directions that are symmetric with respect to the principal directions if and only if $\kappa_{n}(a, b)=\kappa_{n}(\tilde{a}, \tilde{b})$. Therefore, Equation (1) implies

$$
\frac{a^{2} e+2 a b f+b^{2} g}{a^{2} E+2 a b F+b^{2} G}=\frac{\tilde{a}^{2} e+2 \tilde{a} \tilde{b} f+\tilde{b}^{2} g}{\tilde{a}^{2} E+2 \tilde{a} \tilde{b} F+\tilde{b}^{2} G},
$$

or equivalently

$$
\begin{aligned}
& (\tilde{a} b-a \tilde{b})[2 a \tilde{a} E f-2 a \tilde{a} e F+\tilde{a} b E g+a \tilde{b} E g+2 b \tilde{b} F g \\
& -(\tilde{a} b e+a \tilde{b} e+2 b \tilde{b} f) G]=0 .
\end{aligned}
$$

As $\tilde{a} b-a \tilde{b} \neq 0$ we immediately obtain equivalence to Equation (4).

On the other hand if $s, t$ are linearly dependent and symmetric with respect to the principal directions, this direction must be principal. Since principal directions are eigenvectors of the shape operator, they fulfill

$$
\left(\begin{array}{ll}
E & F \\
F & G
\end{array}\right)^{-1}\left(\begin{array}{ll}
e & f \\
f & g
\end{array}\right)\left(\begin{array}{l}
a \\
b
\end{array}\right)=\lambda\left(\begin{array}{l}
a \\
b
\end{array}\right)
$$

Eliminating $\lambda$ yields

$$
a^{2}(e F-E f)+a b(e G-E g)+b^{2}(f G-F g)=0,
$$

which is precisely Equation (4) for $(\tilde{a}, \tilde{b})=(a, b)$.
Lemma 2.2. Apart from umbilical points an S-net is the same as a net with equal normal curvature in both directions.

Proof. The Symmetry of two tangent vectors $s, t$ implies equal normal curvature according to Corollary 2.6. The angle between $s$ [resp. $t$ ] and the first principal curvature direction is denoted by $\alpha$ [resp. $\beta$ ]. If $\kappa_{1} \neq \kappa_{2}$ then $\kappa_{n}(t)=\kappa_{n}(s)$ implies that $t$ and $s$ are either linearly dependent or symmetric with respect to the principal directions. This follows immediately from Euler's formula:

$$
\begin{aligned}
& \kappa_{1} \cos ^{2} \alpha+\kappa_{2} \sin ^{2} \alpha=\kappa_{1} \cos ^{2} \beta+\kappa_{2} \sin ^{2} \beta \\
\Rightarrow & \left(\kappa_{1}-\kappa_{2}\right) \cos ^{2} \alpha=\left(\kappa_{1}-\kappa_{2}\right) \cos ^{2} \beta \\
\Rightarrow & \beta \in\{\alpha,-\alpha, \pi+\alpha, \pi-\alpha\},
\end{aligned}
$$

and thus, $t$ and $s$ are linearly dependent $(\beta \in\{\alpha, \pi+\alpha\})$ or symmetric to the principal directions $(\beta \in\{-\alpha, \pi-\alpha\})$.

LemmA 2.12. Let $A B C$ be a triangle. Then $\frac{B-A}{\|B-A\|^{2}}-\frac{C-A}{\|C-A\|^{2}}$ is a tangent vector to the circumcircle of $A B C$ at $A$.

Proof. Let us consider the unit circle $c$ around $A$. Reflecting a point $X$ in that circle $c$ computes to $\frac{X-A}{\|X-A\|^{2}}$. Thus, reflecting $A, B$ in this circle yields vectors $\frac{B-A}{\|B-A\|^{2}}$ and $\frac{C-A}{\|C-A\|^{2}}$. Reflecting the circumcircle of $A B C$ in circle $c$ reflects it to a straight line as the circumcircle of $A B C$ passes through the center (which is $A$ ) of the circle we are reflecting

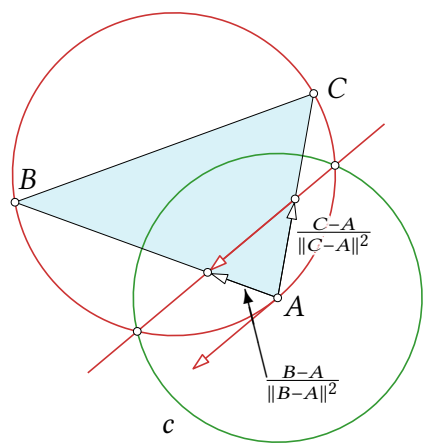
in. And the center is reflected to infinity. Additionally, the reflected straight line is parallel to the tangent at $A$ as it passes through the two fixed points which consist of the intersection points of the two involved circles. Thus subtracting the two vectors, i.e., $\frac{B-A}{\|B-A\|^{2}}-\frac{C-A}{\|C-A\|^{2}}$, yields a vector parallel to the tangent at $A$.

Proof of Equation (5). We have to show that $v-p$ is parallel to $\left\langle q_{1}-p, n_{1}\right\rangle n \times n_{2}+\left\langle q_{2}-p, n_{2}\right\rangle n_{1} \times n$.

The tip of the cone $v$ is contained in all three planes which implies

$$
\langle v-p, p\rangle=0, \quad\left\langle v-q_{1}, n_{1}\right\rangle=0, \quad\left\langle v-q_{2}, n_{2}\right\rangle=0 .
$$

To show parallelity of the two vectors we show that their cross product vanishes:

$$
\begin{aligned}
& (v-p) \times\left(\left\langle q_{1}-p, n_{1}\right\rangle n \times n_{2}+\left\langle q_{2}-p, n_{2}\right\rangle n_{1} \times n\right) \\
= & \left\langle q_{1}-p, n_{1}\right\rangle\left(\langle n, v-p\rangle n_{2}-\left\langle n_{2}, v-p\right\rangle n\right) \\
+ & \left\langle q_{2}-p, n_{2}\right\rangle\left(\left\langle n_{1}, v-p\right\rangle n-\langle n, v-p\rangle n_{1}\right) \\
\stackrel{(16)}{=} & \left\langle q_{2}-p, n_{2}\right\rangle\left\langle n_{1}, v-p\right\rangle n-\left\langle q_{1}-p, n_{1}\right\rangle\left\langle n_{2}, v-p\right\rangle n \\
= & \left\langle\left\langle q_{2}-p, n_{2}\right\rangle n_{1}-\left\langle q_{1}-p, n_{1}\right\rangle n_{2}, v-p\right\rangle n \\
\stackrel{(16)}{=} & \left\langle\left\langle q_{2}-p+v-q_{2}, n_{2}\right\rangle n_{1}-\left\langle q_{1}-p+v-q_{1}, n_{1}\right\rangle n_{2}, v-p\right\rangle n \\
= & \left\langle\left\langle v-p, n_{2}\right\rangle n_{1}-\left\langle v-p, n_{1}\right\rangle n_{2}, v-p\right\rangle n \\
= & \left\langle(v-p) \times\left(n_{2} \times n_{1}\right), v-p\right\rangle n=0 .
\end{aligned}
$$

